\title{
Explaining the Spread in Global Mean Thermosteric Sea Level Rise in CMIP5 Climate Models*
}

\author{
Angélique Melet And Benoit MeyssignaC \\ LEGOS/CNRS/CNES/IRD, Université Paul Sabatier, Toulouse, France
}

(Manuscript received 16 March 2015, in final form 25 September 2015)

\begin{abstract}
The ocean stores more than $90 \%$ of the energy excess associated with anthropogenic climate change. The resulting ocean warming and thermal expansion are leading contributors to global mean sea level (GMSL) rise. Confidence in projections of GMSL rise therefore depends on the ability of climate models to reproduce global mean thermosteric sea level (GMTSL) rise over the twentieth century. This study first compares the GMTSL of the climate models from phase 5 of the Coupled Model Intercomparison Project (CMIP5) to observations over 1961-2005. Although the model ensemble mean is within the uncertainty of observations, the model ensemble exhibits a large spread. The authors then aim to explain the spread in CMIP5 climate model GMTSL over the twentieth and twenty-first centuries. It is shown that the climate models' GMTSL rise depends linearly on the time-integrated radiative forcing $F$ (under continuously increasing radiative forcing). The constant of proportionality $\mu$ expresses the transient thermosteric sea level response of the climate system, and it depends on the fraction of excess heat stored in the ocean, the expansion efficiency of heat, the climate feedback parameter, and the ocean heat uptake efficiency. The across-model spread in $\mu$ explains most $(>70 \%)$ of the across-model spread in GMTSL rise over the twentieth and twenty-first centuries, while the across-model spread in timeintegrated $F$ explains the rest. The time-integrated $F$ explains less variance in the across-model GMTSL rise in twenty-first-century than in twentieth-century simulations, as the spread in $F$ is reduced over the twenty-first century because the anthropogenic aerosol forcing, which is a large source of uncertainty in $F$, becomes relatively smaller.
\end{abstract}

\section{Introduction}

Sea level rise is one of the most adverse consequences of climate change. It threatens millions of people who are living on low-lying islands or close to the coastline and has both direct and indirect socioeconomic impacts, which appear to be overwhelmingly negative (Wong et al. 2013). For this reason it is essential to understand why current sea level is rising and how fast it will rise in the next decades to centuries in response to climate change.

At the global scale, sea level has been rising during the twentieth century as a consequence of two main processes: 1) the expansion of the ocean as it warms up

\footnotetext{
* Supplemental information related to this paper is available at the Journals Online website: http://dx.doi.org/10.1175/JCLI-D-150200.s1.

Corresponding author address: Angélique Melet, Geophysical Fluid Dynamics Laboratory, Princeton University, Princeton Forrestal Campus, 201 Forrestal Road, Princeton, NJ 08540-6649. E-mail: angelique.melet@legos.obs-mip.fr
}

and 2) the increase of the ocean mass as glaciers and ice sheets lose mass and add more water to the ocean. Sea level observations from tide gauges indicate that global mean sea level (GMSL) has been rising at a rate of $2.0 \pm$ $0.3 \mathrm{~mm} \mathrm{yr}^{-1}$ since 1971 (Church et al. 2013a). In situ ocean temperature observations, which have a quasiglobal coverage since 1971 (except in the abyssal ocean and in the Southern Ocean; Abraham et al. 2013), show that most of this rise is explained by the thermal expansion of the ocean. Indeed, the thermal expansion of the ocean contributed $40 \%$ of GMSL rise over $1971-2010\left(0.8 \pm 0.3 \mathrm{~mm} \mathrm{yr}^{-1}\right)$, while over the same period land glacier ice melt contributed $31 \%$ $\left(0.6 \pm 0.4 \mathrm{~mm} \mathrm{yr}^{-1}\right)$ and ice sheet contribution was probably close to zero (Church et al. 2013a; Gregory et al. 2013b). Since the 1990s, tide gauge records and satellite altimetry show that GMSL has been accelerating to the rate of $3.2 \mathrm{~mm} \mathrm{yr}^{-1}$ (Ablain et al. 2014). This acceleration is essentially due to a slight increase of the ocean thermal expansion contribution, up to $1.1 \pm 0.3 \mathrm{~mm} \mathrm{yr}^{-1}$ over the $1993-2010$ period, and to a significant increase of the ice sheet contribution, up to 
$0.6 \pm 0.2 \mathrm{~mm} \mathrm{yr}^{-1}$ over the same period (Church et al. 2013a; Gregory et al. 2013b).

Climate change is expected to continue over the next century in response to more greenhouse gas (GHG) emissions from anthropogenic sources. As a consequence, GMSL is expected to rise faster and higher in the future. Atmosphere-ocean coupled general circulation models (which we henceforth refer to as climate models) from phase 5 of the Coupled Model Intercomparison Project (CMIP5) indicate an increase of the GMSL by several tens of centimeters over the twenty-first century for any of the four future GHG emissions scenarios (viz., RCP2.6, RCP4.5, RCP6.0, and RCP8.5; Moss et al. 2010). They also indicate that the primary contributor to sea level rise over the twentyfirst century will still be the thermal expansion of the ocean (with a contribution of $35 \%, 40 \%, 40 \%$, and $44 \%$ of the total GMSL rise for the RCP scenarios RCP2.6, RCP4.5, RCP6.0, and RCP8.5, respectively; Church et al. 2013a). Yet, climate model projections of future sea level remain uncertain. For any given RCP scenario, climate model projections of future GMSL over the period 2081-2100 show a spread of $\pm 32 \%$ around the climate model ensemble mean (Church et al. 2013a). This large spread prevents making accurate and reliable sea level rise projections as needed for impact studies.

Part of the spread in the GMSL across models using the same RCP scenario can be explained by the internal variability of the coupled climate system that is spontaneously generated in the absence of changes in the external forcing of the climate system (such as changes in the radiative forcing due to changes in concentrations of radiatively active gases and aerosols or in solar irradiance). Indeed, internal climate variability has a different phasing in the different coupled models, which can lead to different variability in GMSL projections. However, at multidecadal time scales, this unforced variability in GMSL is very small (it oscillates around $0 \pm 1 \mathrm{~mm} \mathrm{yr}^{-1}$ at decadal time scales; Cazenave et al. 2014) and can certainly not explain the spread of tens of $\mathrm{cm}$ in GMSL projections over 2081-2100 (Church et al. 2013a). Actually, most of the spread in GMSL projections comes from the forced variability in GMSL (i.e., the variability in response to the external radiative forcing agents, including volcanic and anthropogenic aerosols, greenhouse gases, and solar irradiance). The radiative forcing due to a perturbation in a forcing agent (e.g., perturbation in the concentration of a radiatively active gas) is defined by the change in global mean net downward radiation at the tropopause caused by the perturbation. In contrast to the unforced internal variability, the forced variability is supposed to be the same across climate model simulations of the same scenario because it responds to the same prescribed external radiative forcing. However, it turns out to be different from one model to another because the physical and dynamical processes driving the forced variability in sea level are not well understood and inaccurately represented in climate models. Therefore, different climate models give significantly different estimates of the forced response of the GMSL, causing a large spread in GMSL projections whatever the scenario considered. Unlike the spread due to the internal variability of the climate system, the spread in the forced response of the GMSL could be reduced with a better understanding of the processes that drive the forced response of GMSL.

In this paper, we focus on the GMSL rise resulting from the thermal expansion of the ocean [also called global mean thermosteric sea level (GMTSL)] because it is the main contributor to present-day sea level rise and it should play a primary role in twenty-first-century sea level rise. As for GMSL, projections of future GMTSL over 2081-2100 show a large spread around the climate model ensemble mean $( \pm 23 \%$ spread for all RCP scenarios), which is mainly due to differences in the GMTSL forced response to future radiative forcing in climate models. The main objective of this paper is to explain the spread in GMTSL rise among climate models based on a better understanding of how GMTSL responds to radiative forcing and by identifying potential ways to reduce the spread across models.

We analyze results from 33 CMIP5 models. We used outputs from the twentieth-century and twenty-firstcentury (scenarios RCP4.5 and RCP8.5) simulations to quantify the differences in past and projected GMTSL variations among climate models (section 1$)$. We compare the past GMTSL variations from twentieth-century simulations with observations to evaluate to what extent each climate model reproduces the observed GMTSL rise of the past decades (section 2). We then develop a simple relationship linking GMTSL variations to changes in the radiative forcing of the climate system based on the energy balance of the climate system (section 3). This relationship shows that at interannualto-centennial time scales, under continuously increasing forcing, GMTSL rise is approximately proportional to the time-integrated radiative forcing of the climate system $F$. It also shows that the constant of proportionality essentially depends on two key climate parameters: the climate feedback parameter $\alpha$ and the ocean heat uptake efficiency $\kappa$ (section 3$)$. We check that this relationship is verified in climate model simulations of twentieth- and twenty-first-century climate change, and we evaluate to what extent the spread in $F$ and in key climate parameters among models can explain the spread in GMTSL rise. 


\section{Thermal expansion of the ocean from climate models}

\section{a. Climate models}

Climate models considered in this study are atmosphereocean coupled general circulation models (AOGCMs) and earth system models (ESMs, which expand on AOGCMs to include representation of the carbon cycle) from the CMIP5 archive (http://cmip-pcmdi.llnl.gov; Taylor et al. 2012). The first ensemble member (r1i1p1) for historical and pre-industrial control (piControl) experiments of each model were downloaded in spring 2014. Historical simulations refer to experiments simulating climate change since the mid-nineteenth century by prescribing time-dependent atmospheric composition, especially regarding anthropogenic greenhouse gases and aerosols. PiControl simulations provide the reference state of historical simulations; they are equivalent to historical simulations but prescribe a constant atmospheric composition, corresponding to preindustrial conditions. The piControl and historical simulations are initialized by the same spunup state, provided by a spinup run of hundreds to thousands of years using a constant, preindustrial atmospheric composition, which aims at equilibrating the model. The selection of CMIP5 models for the calculation of thermal expansion was based on the availability of $3 \mathrm{D}$ temperature and salinity fields in the historical and piControl experiments, resulting in a total of 33 models. Projections over the twenty-first century from the CMIP5 RCP4.5 and RCP8.5 scenarios for the concentrations of aerosols and greenhouse gases (leading to a radiative forcing of $4.5 \mathrm{~W} \mathrm{~m}^{-2}$ at stabilization after 2100 for RCP4.5 and to a radiative forcing greater than $8.5 \mathrm{~W} \mathrm{~m}^{-2}$ in 2100 for RCP8.5) are also analyzed for the subset of models for which needed data were available.

\section{b. Calculation}

The monthly 3D temperature and salinity fields of the CMIP5 models were first averaged annually. The annual potential temperature fields were then converted into in situ temperature fields [using the methodology of Bryden (1973)].

Thermal expansion over the full ocean depth is needed for the calculation of the thermosteric component of sea level rise. To allow an evaluation of the thermal expansion simulated by the climate models over the instrumental period (section 2c), we also consider thermal expansion over 0-700-m depth in addition to the full ocean depth thermal expansion. Indeed, observational estimates of thermal expansion are more accurate and sometimes available only for the 0-700-m layer (observations of the ocean in situ temperature and salinity are sparse below $700 \mathrm{~m}$, especially before the 2000 s and the deployment of ARGO floats; Abraham et al. 2013).

Thermal expansion has then been computed from the annual 3D in situ temperature and salinity fields using the United Nations Educational, Scientific and Cultural Organization (UNESCO) 1980 international equation of state (IES80). A global average over the ocean was computed for each model on its own grid, excluding the marginal seas and lakes (such as the Mediterranean Sea, Red Sea, Black Sea, Caspian Sea, Baltic Sea, Persian Gulf, Hudson Bay, and Great Lakes) where the simulated temperature and salinity fields are less reliable.

Most spinup integrations of climate models are shorter than the characteristic simulation length needed for the deep ocean to reach quasi-equilibrium (which is thousands of years). As a consequence, most historical and piControl simulations, which are initialized with the spinup integration, are still drifting, in particular in the deepest layers. As thermosteric sea level is a depthintegrated variable, the long-term climate drift can have a large effect on this variable in climate models and has to be removed from the historical simulation (Sen Gupta et al. 2013). To avoid capturing natural and physical oscillations, the long-term climate drift is estimated by a low-order polynomial fit of the piControl run over the period corresponding to the historical simulation. Here, a quadratic fit of the global mean thermal expansion was calculated for each model and each reference level. The global mean thermal expansion of each model is then detrended by removing the corresponding long-term drift. We tested the sensitivity of GMTSL changes to using the full piControl simulations to detrend the historical GMTSL [using the method of Sen Gupta et al. (2013)] and found that the differences in the model ensemble GMTSL mean and standard deviation induced by the detrending methods are negligible (smaller by one order of magnitude than the GMTSL changes we are analyzing over the historical period; not shown). Because of the detrending, the zero of GMSL is arbitrary, and we chose to reference our estimates in 2005.

\section{c. Comparison with observations of the global mean steric sea level}

Two different periods are considered for evaluating the ability of individual CMIP5 models to reproduce the observed sea level rise. As most CMIP5 historical simulations end in 2005, we will consider the 1900-2005 period for the twentieth century. To better constrain the assessment of climate models' ocean thermal expansion, we also consider the 1961-2005 period, since the amount of observations have significantly increased since the 1960s (Abraham et al. 2013). 
a)

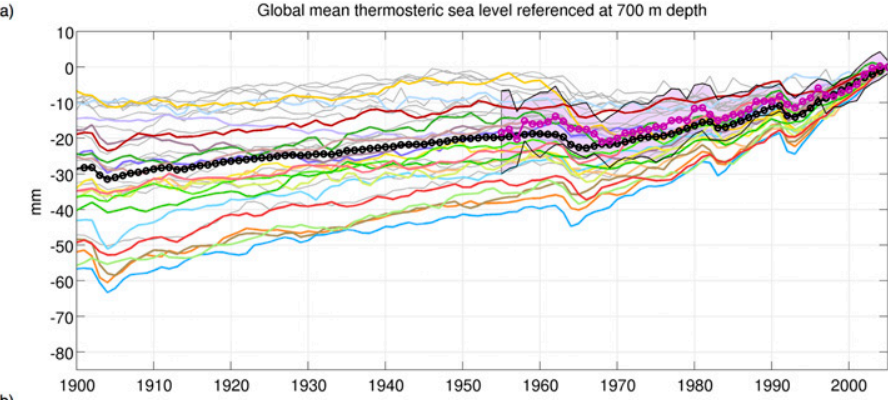

b)

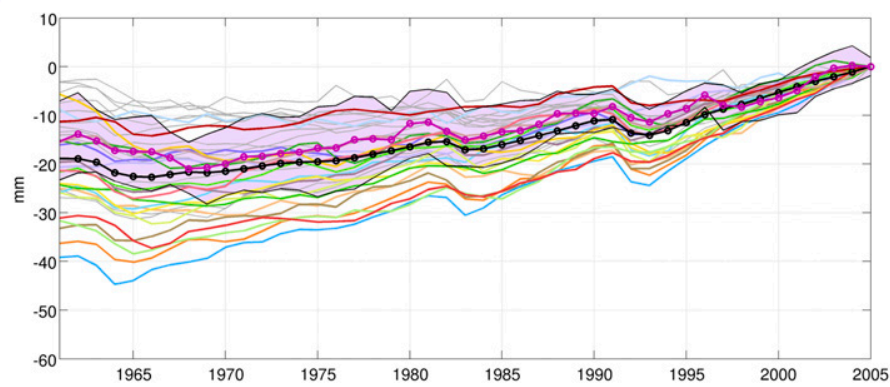

c)

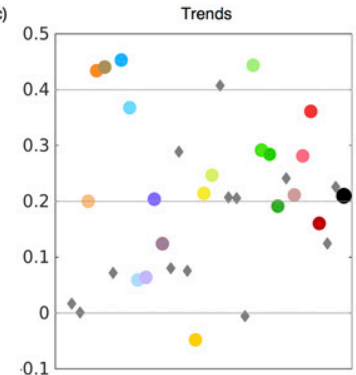

d)

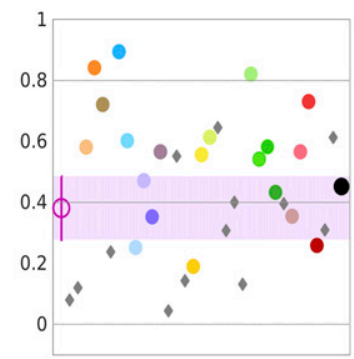

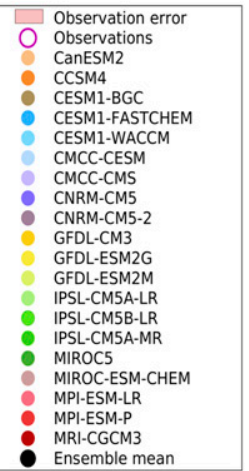

FIG. 1. Thermosteric sea level (mm) referenced in 2005 for the 0-700-m layer over (a) 1900-2005 and (b) 1961-2005. Observational estimates are the mean of Levitus et al. (2009), Ishii and Kimoto (2009), and Domingues et al. (2008). An uncertainty related to the observational estimates is shown as the purple shaded area. Thermosteric sea level trend $\left(\mathrm{mm} \mathrm{yr}^{-1}\right)$ is shown for the $0-700$ - $\mathrm{m}$ layer over (c) 1900-2005 and (d) 1961-2005. In (c) and (d), spacing along the $x$ axis is used to separate the different climate models (the model ensemble mean is shown as a black dot at the right end of the $x$ axis). The color scheme is identical in each panel: CMIP5 models used in section 3 are shown with thick color lines in (a) and (b) and dots in (c) and (d), while remaining CMIP5 models are shown with gray lines and diamonds.

\section{1) THERMAL EXPANSION OVER 0-700 M}

Thermal expansion over the 0-700-m layer simulated by CMIP5 models over 1900-2005 and 1961-2005 is shown in Fig. 1 for the 33 available models. The thermal expansion of the 20 models used in section 3 (models for which the calculation of the fraction of Earth's energy imbalance stored in the ocean is possible and the result is reasonable; see section 3 ) is plotted in thick color lines. These 20 climate models are well distributed over the spread of all CMIP5 models (gray lines) and are therefore representative of the ensemble of CMIP5 models.

Before the 1960s, no observational estimate of the ocean global mean thermal expansion is available. Since 1961, observations of ocean temperature have become more and more available, providing a quasi-global coverage of the ocean. Thermal expansions based on the observational datasets of Levitus et al. (2009), Ishii and Kimoto (2009), and Domingues et al. (2008) were computed over the 1961-2005 period. We use the error estimates of Llovel et al. (2013), which are calculated from the Levitus et al. (2009) errors of temperature data included in their dataset. We chose a $2 \sigma$ error level $(95 \%$ confidence interval) for the thermal expansion of Levitus et al. (2009), Ishii and Kimoto (2009), and Domingues et al. (2008) and considered the largest envelope to define the uncertainty of the observed thermal expansion (Fig. 1, pink shaded area). Note that formal errors in the mapping methods have been omitted, which likely result in an underestimate of the uncertainty envelope. The observational estimate of the rate of thermal expansion is computed as the trend of the linear regression to the average thermal expansion time series of Levitus et al. (2009), Ishii and Kimoto (2009), and Domingues et al. (2008). The uncertainty of the trend of observed thermal expansion is evaluated using a Monte Carlo methodology. We define 10000 random time series by sampling a normal distribution centered on the mean observed thermal expansion time series of Levitus et al. (2009), Ishii and Kimoto (2009), and Domingues et al. (2008) and with a standard deviation corresponding to the standard deviation of the observed thermal expansion uncertainty. The uncertainty of the trend of thermal expansion is then defined as 3 times the standard deviation of the normal distribution of the trends of the sampled time series (corresponding to the $99 \%$ confidence interval). Note that in this calculation autocorrelations in the random time series were not taken into account, which tends to underestimate the uncertainty on the trend. To compensate for that underestimation, we chose a conservative $99 \%$ confidence interval. 
a)

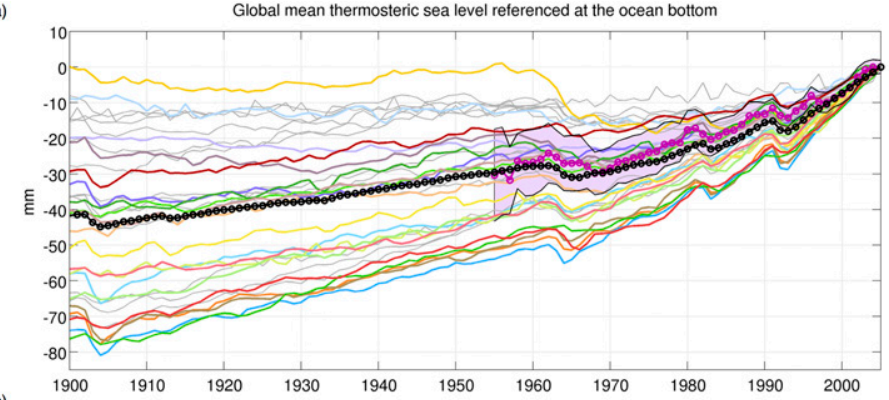

b)

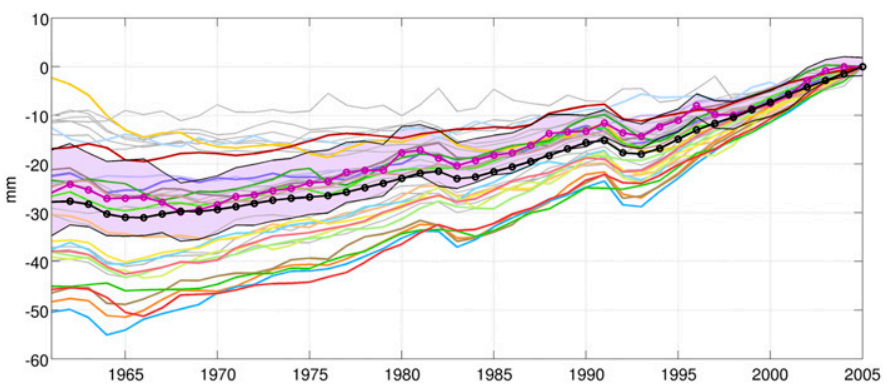

c)

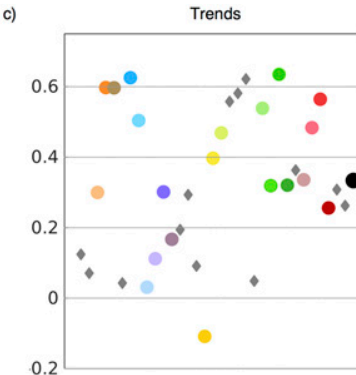

d)

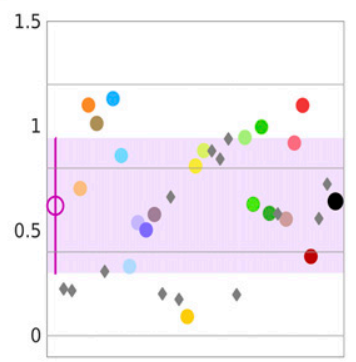

FIG. 2. Thermosteric sea level (mm) referenced in 2005 for the full ocean depth over (a) 1900-2005 and (b) 1961-2005. Observational estimates are the mean of Levitus et al. (2009), Ishii and Kimoto (2009), and Domingues et al. (2008). An uncertainty related to the observational estimates is shown as the purple shaded area. Thermosteric sea level trend $\left(\mathrm{mm} \mathrm{yr}^{-1}\right)$ is shown for the full ocean depth over (c) 1900-2005 and (d) 1961-2005. In (c) and (d), spacing along the $x$ axis is used to separate the different climate models (the model ensemble mean is shown as a black dot at the right end of the $x$ axis). The color scheme is identical in each panel: CMIP5 models used in section 3 are shown with thick color lines in (a) and (b) and dots in (c) and (d), while remaining CMIP5 models are shown with gray lines and diamonds.

Over 1900-2005, the model ensemble global mean thermal expansion of the upper $700 \mathrm{~m}$ of the ocean is $29 \mathrm{~mm}$ (dotted black line in Fig. 1a), with a rate of $0.21 \mathrm{~mm} \mathrm{yr}^{-1}$. Focusing on the subensemble of 20 models, the lowest thermal expansions are given by GFDL CM3 (7 mm), CMCC-CESM (9 mm), and CMCC-CMS (15 mm), corresponding to a rate of thermal expansion weaker than $0.1 \mathrm{~mm} \mathrm{yr}^{-1}$ (Fig. 1c). The highest thermal expansion is given by CESM1(FASTCHEM) $(57 \mathrm{~mm})$, IPSL-CM5ALR $(56 \mathrm{~mm})$, and CCSM4 $(51 \mathrm{~mm})$, with a rate slightly stronger than $0.4 \mathrm{~mm} \mathrm{yr}^{-1}$ (Fig. 1c).

Over 1961-2005, the observed thermal expansion is $16 \mathrm{~mm}$ (Fig. 1b, pink dotted line), with a rate of $0.38 \pm$ $0.11 \mathrm{~mm} \mathrm{yr}^{-1}$ (Fig. 1d). The model ensemble mean thermal expansion is within the error bars of observations, with a value of $19 \mathrm{~mm}$ and a rate of $0.45 \mathrm{~mm} \mathrm{yr}^{-1}$. The lowest thermal expansions of the 20 subensemble of models are given by GFDL CM3 $(6 \mathrm{~mm})$ and CMCCCESM $(9 \mathrm{~mm})$, corresponding to a rate of thermal expansion of approximately $0.2 \mathrm{~mm} \mathrm{yr}^{-1}$ (Fig. 1d). The highest thermal expansions are given by CESM1(FASTCHEM) $(39 \mathrm{~mm})$, CCSM4 $(36 \mathrm{~mm})$ and CESM1 with biogeochemistry [CESM1(BGC)] $(33 \mathrm{~mm})$, with a rate slightly stronger than $0.8 \mathrm{~mm} \mathrm{yr}^{-1}$. Several other models simulate higher than observationally estimated thermal expansion over 1961-2005 (Fig. 1d).
However, a recent study suggests that observations of thermal expansion might be biased low because of poor observational sampling of the Southern Hemisphere (Durack et al. 2014). It should also be kept in mind that our uncertainty estimate for observed thermal expansion trend is rather conservative.

\section{2) THERMAL EXPANSION FOR THE FULL OCEAN DEPTH}

Thermal expansion over the full ocean simulated by CMIP5 models over 1900-2005 and 1961-2005 is shown in Fig. 2. The 20 models used for the calculation of GMTSL (color lines in Fig. 2) are also representative of the ensemble of CMIP5 models for the full ocean depth.

Because of inadequate deep-ocean sampling over the 1961-2005 period, the contribution of the deep ocean to thermal expansion is uncertain. The observational estimate of the full-depth thermal expansion is calculated by adding linear trends for the contribution of the 7002000-m depth layer and the layer below $2000 \mathrm{~m}$ to the Levitus et al. (2009) estimate of the thermal expansion rate over the 0-700-m depth layer. For the 700-2000-m layer, we use a trend of $0.13 \pm 0.05 \mathrm{~mm} \mathrm{yr}^{-1}$ based on the observational analysis of Levitus et al. (2012) over 19552010. Below $2000 \mathrm{~m}$, we use the trend of $0.11 \pm$ $0.10 \mathrm{~mm} \mathrm{yr}^{-1}$ suggested by Purkey and Johnson (2010) 
over the last decades and apply it to the 1900-2005 and 1961-2005 periods. The uncertainty of the trend of thermal expansion over the full ocean depth is computed using the same methodology as for the 0-700-m layer.

Over 1900-2005, the model ensemble mean global mean thermal expansion is $42 \mathrm{~mm}$ (dotted black line in Fig. 2a), corresponding to a rate of $0.33 \mathrm{~mm} \mathrm{yr}^{-1}$ (Fig. 2c). The lowest thermal expansions are still given by GFDL CM3 $(0 \mathrm{~mm})$ and CMCC-CESM $(9 \mathrm{~mm})$, corresponding to a rate of thermal expansion weaker than $0.1 \mathrm{~mm} \mathrm{yr}^{-1}$ (Figs. 2a,c). The highest thermal expansions are given by IPSL-CM5A-MR $(76 \mathrm{~mm})$ and CESM1(FASTCHEM) $(74 \mathrm{~mm})$, with a rate stronger than $0.6 \mathrm{~mm} \mathrm{yr}^{-1}$.

Over 1961-2005, the observed thermal expansion is $26 \mathrm{~mm}$ (Fig. 2b), with a rate of $0.6 \pm 0.3 \mathrm{~mm} \mathrm{yr}^{-1}$ (Fig. 2d). The model ensemble mean global mean thermal expansion is very close to the observed one, with a value of $28 \mathrm{~mm}$. In terms of trends, most models are within the uncertainty of observations. CCSM4, CESM1(FASTCHEM), and MPI-ESM-P give faster than observed thermal expansion over this period, with a rate higher than $1.0 \mathrm{~mm} \mathrm{yr}^{-1}$, while GFDL CM3 gives slower than observed thermal expansion over this period, with a rate of $0.1 \mathrm{~mm} \mathrm{yr}^{-1}$ (Fig. 2d).

The comparison of modeled thermal expansion over the $0-700-\mathrm{m}$ and full ocean depth with observations shows that the model ensemble mean global mean thermal expansion is within the uncertainty of observations, both for the annual time series and the trends. However, individual models show a substantial spread over the ensemble mean, and a significant number of models simulate thermal expansion that is not within the uncertainty of observations. Some models consistently overestimate thermal expansion over the twentieth century [for instance, CESM1(FASTCHEM) and CESM1(BGC) in our 20-model subensemble], while other models consistently underestimate it (for instance, GFDL CM3 and CMCC-CESM in our 20-model subensemble). Our goal here is not to point out specific models as not able to realistically simulate thermal expansion over the twentieth century but to show that there is a substantial spread across models and that evaluating the ensemble mean is not sufficient. Individual evaluations of models should also be performed.

\section{d. Correction for volcanic forcing}

Gregory et al. (2013a) and Church et al. (2013b) suggested that a correction of $0.1 \mathrm{~mm} \mathrm{yr}^{-1}$ should be applied to the GMTSL for the omission of volcanic forcing in the piControl experiments of some CMIP models. We check here whether this correction should be applied. Explosive volcanic eruptions produce aerosols in the stratosphere that reflect incident solar radiation and weaken the solar radiation at the ocean surface. The climate system adjusts to this time-mean natural negative radiative forcing over long time scales. However, variations of the intensity and frequency of volcanic eruptions cause radiative forcing fluctuations for the climate system; more volcanic activity than is typical of the long term results in a negative radiative forcing and vice versa. Thermal expansion of the ocean is therefore impacted by volcanic forcing. After major volcanic eruptions, the ocean recovers from the cooling caused by the volcanic forcing, and the rate of expansion is enhanced for a few decades (Church et al. 2005; Gregory et al. 2006; Delworth et al. 2005). As most of the climate models used in phase 3 of the Coupled Model Intercomparison Project (CMIP3) omitted volcanic forcing in their spinup and piControl experiments, the imposition of historical volcanic forcing in the historical experiment of some models represented a time-mean negative forcing in the historical experiment relative to the piControl experiment. Gregory (2010) and Gregory et al. (2013a) showed that this resulted in a significant negative bias in ocean heat uptake and an underestimate of thermal expansion (up to $40 \%$ ). To overcome this bias, they suggested adding a correction of $0.1 \pm$ $0.1 \mathrm{~mm} \mathrm{yr}^{-1}$ to the thermal expansion rate of climate models that imposed volcanic forcing in their historical experiment but omitted it in their piControl experiment. Church et al. (2013a) suggested that thermal expansion rates of CMIP5 models may be similarly underestimated, depending on the details of the individual model control runs, and therefore applied the same correction of $0.1 \mathrm{~mm} \mathrm{yr}^{-1}$.

As different background volcanic aerosol levels were chosen across CMIP5 models [zero or an average volcanic forcing background in general; see Table 12.1 of Collins et al. (2013) for more details] and CMIP5 models use different ways of prescribing volcanic forcing than CMIP3 models, we investigated the impact of volcanic forcing on thermal expansion rates in CMIP5 models before applying the same correction of $0.1 \mathrm{~mm} \mathrm{yr}^{-1}$. The general recommendation for the prescription of volcanic forcing in CMIP5 was to either omit it entirely both from the piControl and historical experiments or to prescribe the same background volcanic forcing in both (Collins et al. 2013). However, half the CMIP5 models used in this study for estimating thermal expansion did not follow this guidance.

We split the ensemble of models in two groups: models prescribing volcanic forcing in both their piControl and historical experiments (piC-V group) and models prescribing volcanic forcing only in their historical experiments (non-piC-V group). The time series 
over the historical period of the detrended global mean thermal expansion referenced at the ocean bottom are shown in Fig. 3a for the different models of each group (in green for the 15 models in the piC-V group and in black for the 15 models in the non-piC-V group). Although it is expected from the physical arguments presented in Gregory et al. (2013a) that models of the non-piC-V group would exhibit weaker rates of thermal expansion than models of the piC-V group, no clear separation of the two groups is found in Fig. 3a. This lack of separation is further illustrated when averaging the thermal expansion of the models in the two different groups (Fig. 3b); over the 1961-2005 period, no clear separation is found. In both groups, the global thermal expansion suddenly drops after major volcanic eruptions (such as after 1883 following the Krakatau eruption), but the rate of recovery of the ocean from the volcanic-forced cooling is similar. Moreover, the rate of thermal expansion of the two groups in the 0-700-m layer is mostly consistent with and within the uncertainty of observational estimates of the rate of thermal expansion based on the analysis of Levitus et al. (2009), Ishii and Kimoto (2009), and Domingues et al. (2008) (Figs. 3c,d).

Linear regressions of the differences of thermal expansion between the piC- $\mathrm{V}$ group and non-piC- $\mathrm{V}$ group indicate that the rate of thermal expansion in the nonpiC-V group is slightly stronger $\left(+0.002 \mathrm{~mm} \mathrm{yr}^{-1}\right)$ than the rate of the piC-V group in the upper $700 \mathrm{~m}$ of the ocean and is only slightly weaker when deepest layers of the ocean are considered $\left(-0.02 \mathrm{~mm} \mathrm{yr}^{-1}\right.$ for the topto-bottom ocean over 1961-2005). Therefore, the correction to be applied to the top-to-bottom thermal expansion rate of models in the non-piC-V group would be $+0.02 \mathrm{~mm} \mathrm{yr}^{-1}$ when considering the 1961-2005 period. The amplitude of the correction is within the range of uncertainty of the correction of $0.1 \pm 0.1 \mathrm{~mm} \mathrm{yr}^{-1}$ suggested by Gregory et al. (2013a) but is almost an order of magnitude weaker than the correction of $0.1 \mathrm{~mm} \mathrm{yr}^{-1}$ applied in Church et al. (2013a,b). Applying a correction of $0.1 \mathrm{~mm} \mathrm{yr}^{-1}$ to the thermal expansion rate of the non-piC-V models would therefore result in a larger thermal expansion rate of the non-piC-V group than of the piC-V group (which is contradictory to the purpose of the correction in the first place).

Given the spread of the thermal expansion in piC-V and non-piC-V models (Fig. 3a) and the lack of separation between the two groups, we consider that differences in thermal expansion between the piC- $\mathrm{V}$ and non-piC- $\mathrm{V}$ groups are not a signature of different volcanic forcing protocols but rather the signature of the spread of models due to other sources of uncertainty.
Although correcting the rate of thermal expansion of models omitting volcanic forcing in their piControl experiments is based on physical arguments (Gregory et al. 2013a), the lack of separation between the thermal expansion simulated by non-piC-V and piC-V CMIP5 models suggests that the tuning of CMIP5 nonpiC-V models to reproduce key climate metrics (such as the sea surface temperature) could have compensated the impact of the omission of volcanic forcing in their piControl experiments. Indeed, time series of the nearsurface air temperature of the piC-V and non-piC-V groups do not exhibit a clear separation either (not shown), which supports the hypothesis that the calibration of various parameters of the models compensates the impact of prescribing or not prescribing volcanic forcing in piControl experiments.

Finally, we stress the fact that correcting models for volcanic forcing is not straightforward, as already highlighted in Gregory et al. (2013a). The correction should be model dependent, as volcanic forcing is prescribed differently in CMIP5 models and models have different sensitivities to volcanic forcing. The choice of the background level of volcanic forcing to be applied in the spinup, piControl, and historical experiments (in between major explosive eruptions) is not simple. The background volcanic forcing prescribed in the historical experiment is zero in some models, is the same as in the piControl experiment in others, or is below that of the piControl experiment (Table 12.1 of Collins et al. 2013). It is also unclear what correction should be applied to different layers of the ocean (i.e., when comparing to observations in the upper ocean), as volcanic forcing is expected to cause a larger discrepancy in the deep rather than the upper ocean because deeper layers adjust on longer time scales (Gregory et al. 2013a).

Based on the previous analysis, we decided to apply no correction to the thermal expansion rates simulated by models omitting volcanic forcing in their piControl experiments.

\section{Understanding the spread in CMIP5 model thermal expansion}

\section{a. Fraction of energy stored in the ocean}

Because of increased atmospheric GHG and aerosols concentrations over the last century due to human activities, incoming shortwave radiation received from the sun $\left(\mathrm{SW}_{\mathrm{in}}\right)$ exceeds the sum of shortwave radiation reflected by Earth $\left(\mathrm{SW}_{\text {out }}\right)$ and of longwave radiation emitted by the surface and atmosphere ( $\left.\mathrm{LW}_{\text {out }}\right)$ at the top of the atmosphere (Church et al. 2013a). As a result, the top-of-the-atmosphere (TOA) radiative budget $N$ is 
a)Top-to-bottom
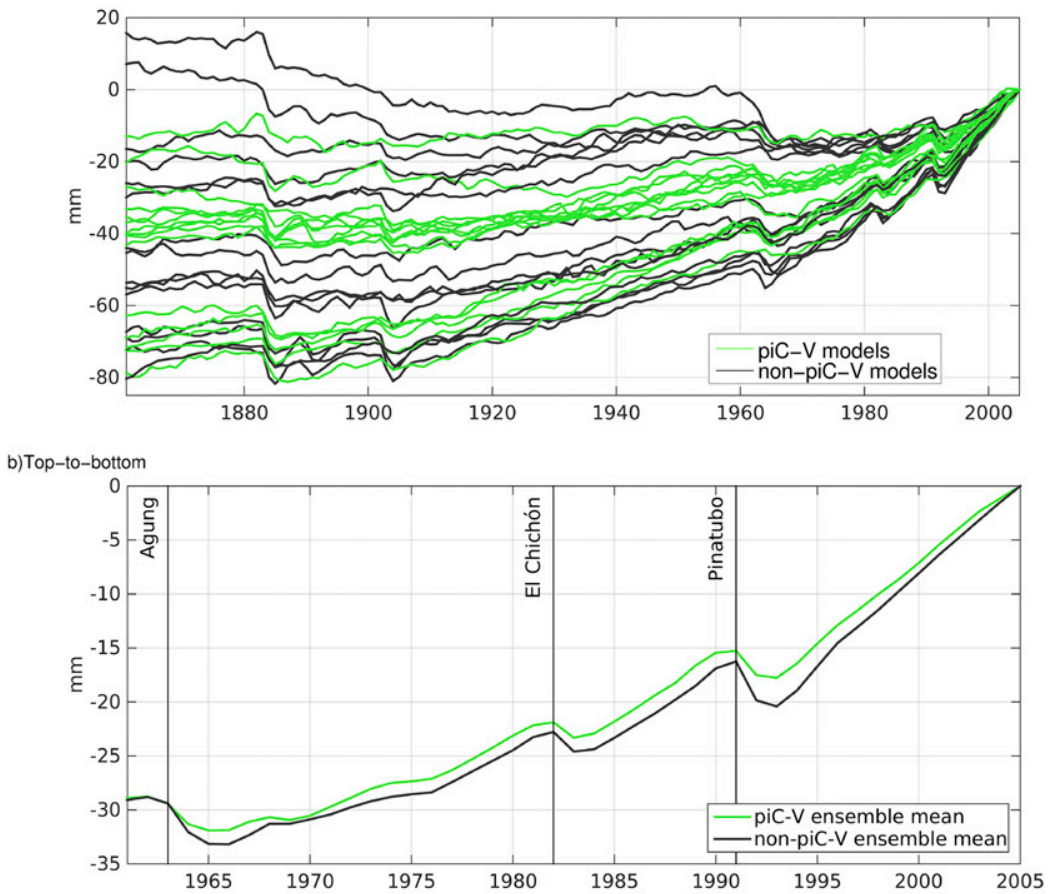

c) $0-700 \mathrm{~m}$

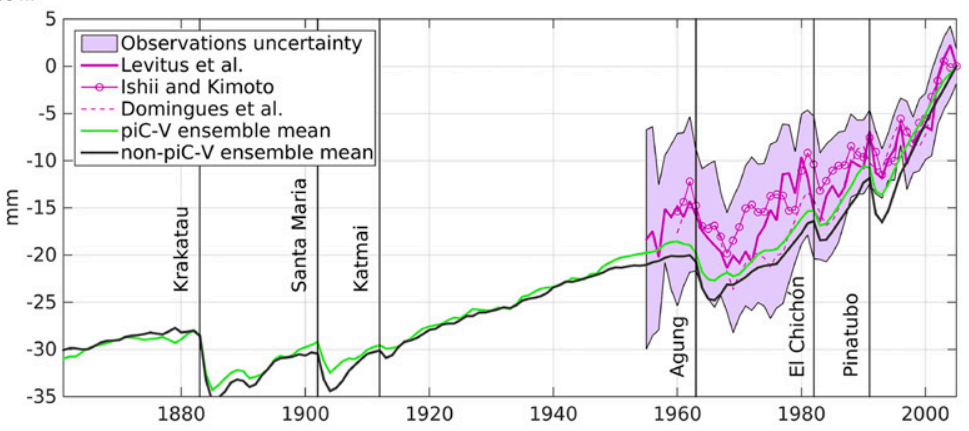

d) $0-700 \mathrm{~m}$

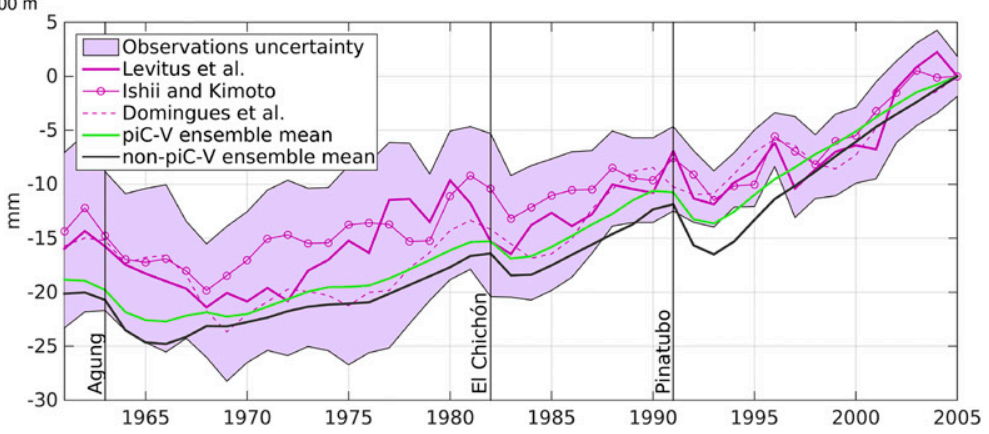

FIG. 3. (a) Global mean top-to-bottom thermosteric sea level (referenced to year 2005). Models prescribing volcanic forcing in the piControl experiment (piC-V group) are shown in green, while models omitting volcanic forcing in the piControl experiment (non-piC-V group) are shown in black (see Table 1). (b) Ensemble global mean top-to-bottom thermosteric sea level referenced in 2005 for models prescribing volcanic forcing in the piControl experiment (piC-V group; green) and for models omitting volcanic forcing in the piControl experiment (non-piC-V group; black). (c) As in (b), but for the 0-700-m global mean thermosteric sea level. (d) Zoomed-in view of (c) over the 1961-2005 period. In (c) and (d), the observational estimates of Levitus et al. (2009), Ishii and Kimoto (2009), and Domingues et al. (2008) are shown with purple lines. An uncertainty related to the observational estimates is shown as the purple shaded area. The years and names of major volcanic eruptions are indicated with black vertical lines. 
unbalanced and energy accumulates in the earth system, essentially in the form of heat following this equation:

$$
N=\mathrm{SW}_{\text {in }}-\left(\mathrm{SW}_{\text {out }}+\mathrm{LW}_{\text {out }}\right) .
$$

Since the ocean has a much greater heat capacity than the atmosphere, the land, and the cryosphere, it stores most of this excess of energy (Levitus et al. 2005; Church et al. 2011).

On interannual to longer time scales, the fraction of total Earth energy excess that is stored in the ocean $\beta$ exceeds $80 \%$ (Church et al. 2013a; Palmer and McNeall 2014). This fraction of energy is defined as $\beta=$ GOHU/ TE, where GOHU is the globally integrated ocean heat uptake (i.e., the global ocean heat content increase in joules) and TE is the total energy excess in the earth system (joules), corresponding to the time- and surfaceintegrated global mean net TOA radiative flux $N$ (watts per meter squared). Therefore, the total heat (joules) that enters the ocean between $t_{\text {ref }}$ and $t$ under a radiative imbalance $N(t)$ is given by the following relation:

$$
\mathrm{GOHU}_{t_{\mathrm{ref}}, t}=\beta S \int_{t_{\mathrm{ref}}}^{t} N(\tau) d \tau,
$$

where $S$ is the surface area of Earth (meters squared; note that $S$ should be the surface area of Earth at the TOA, which is roughly $2 \%$ larger) and $t_{\text {ref }}$ is the year of reference for the computation of GOHU. We chose year 1900 for $t_{\text {ref }}$ in historical runs and year 2006 for $t_{\text {ref }}$ in twenty-first-century projections.

We test whether this linear relationship holds in climate model simulations of the twentieth and twenty-first centuries. The variable $\beta$ is calculated by ordinary least squares (OLS) regression of GOHU against the timeintegrated time series of $N$. Based on the availability of CMIP5 data, the calculation of $\beta$ was possible for 24 models for the twentieth century and for 14 models for the RCP8.5 twenty-first-century projection (see Figs. 4 and 5; Table 1). To remove the potential long-term model drift, $N$ is detrended by subtracting the linear regression of $N$ over the piControl segment parallel to the period considered (historical or projection). GOHU is also corrected for the long-term model drift consistently with the correction applied to $N$. Thus, following (2), a quadratic fit of GOHU over the parallel piControl segment is subtracted. GOHU decreases during years following major volcanic eruptions (Church et al. 2005; Gregory et al. 2013b). Since our interest here is in the long-term (from multidecadal to multicentennial) response of GOHU to the radiative forcing, we excluded years affected by volcanic eruptions before computing $\beta$ (and in the following analyses). Church et al. (2005) showed that the impact of large volcanic eruptions on global ocean heat content is characterized by a rapid reduction in global ocean heat content during the year following the eruption followed by a period of recovery of a few years when global ocean heat content increases faster than before the eruption. Following Church et al. (2005), we chose to remove from our time series the five years following each large volcanic eruption (including Krakatau in 1883, Santa Maria in 1902, Mount Katmai in 1913, Mount Agung in 1963, El Chichón in 1982, and Mount Pinatubo in 1991). Note, however, that this ad hoc criterion does not remove the long-term effect of volcanic forcing (background volcanic forcing and long-term effect of large eruptions; see Gregory 2010).

In all simulations of the twentieth and twenty-first centuries and for all models, the correlation coefficient for annual mean GOHU with annual mean $\int N d t$ exceeds 0.90 (Figs. 4 and 5), indicating that linearity in (2) is a very good assumption. However, $\beta$ varies across models as the magnitude of GOHU in a given model depends on the latitudes and depths where the heat is stored. This pattern of heat storage depends on the model but not on the scenario for a given model (Kuhlbrodt and Gregory 2012). Consequently, $\beta$ varies across models (range of $\pm 20 \%$ across models) but not across scenarios for a given model (range of $\pm 5 \%$ across scenarios). We will consider $\beta$ as constant with time over $1900-2100$ in the following.

The value of $\beta$ can be estimated from the observational studies of Levitus et al. (2005) (considering the 0-2000-m depth range over 1955-2005), from which we infer that $\beta=0.85$, and of Church et al. (2011) (for the full ocean depth over 1972-2008), from which we infer that $\beta=0.93$. In most climate models, $\beta$ is within $20 \%$ of 0.93 (Fig. 4), which is consistent with observations (note that although a value of $\beta$ greater than 1 physically implies that more energy is stored in the ocean than available in the earth system, we allow values of $\beta$ slightly greater than 1 to take into account potential impacts on $\beta$ of the detrending procedure and of the exclusion of marginal seas). Only some models (MIROC-ESM, FGOALS-g2, NorESM1-M, and NorESM1-ME) have values of $\beta$ that are out of this range, suggesting some energy conservation issues in these models. For this reason they were discarded in following analyses.

\section{b. Thermal expansion of the ocean}

The increase in ocean heat content causes sea level rise through the thermal expansion of seawater. The ratio of global sea level rise due to thermal expansion $\Delta$ GMTSL to the global ocean heat uptake defines the expansion efficiency of heat $\epsilon$ (meters per joule; Russell et al. 2000): 

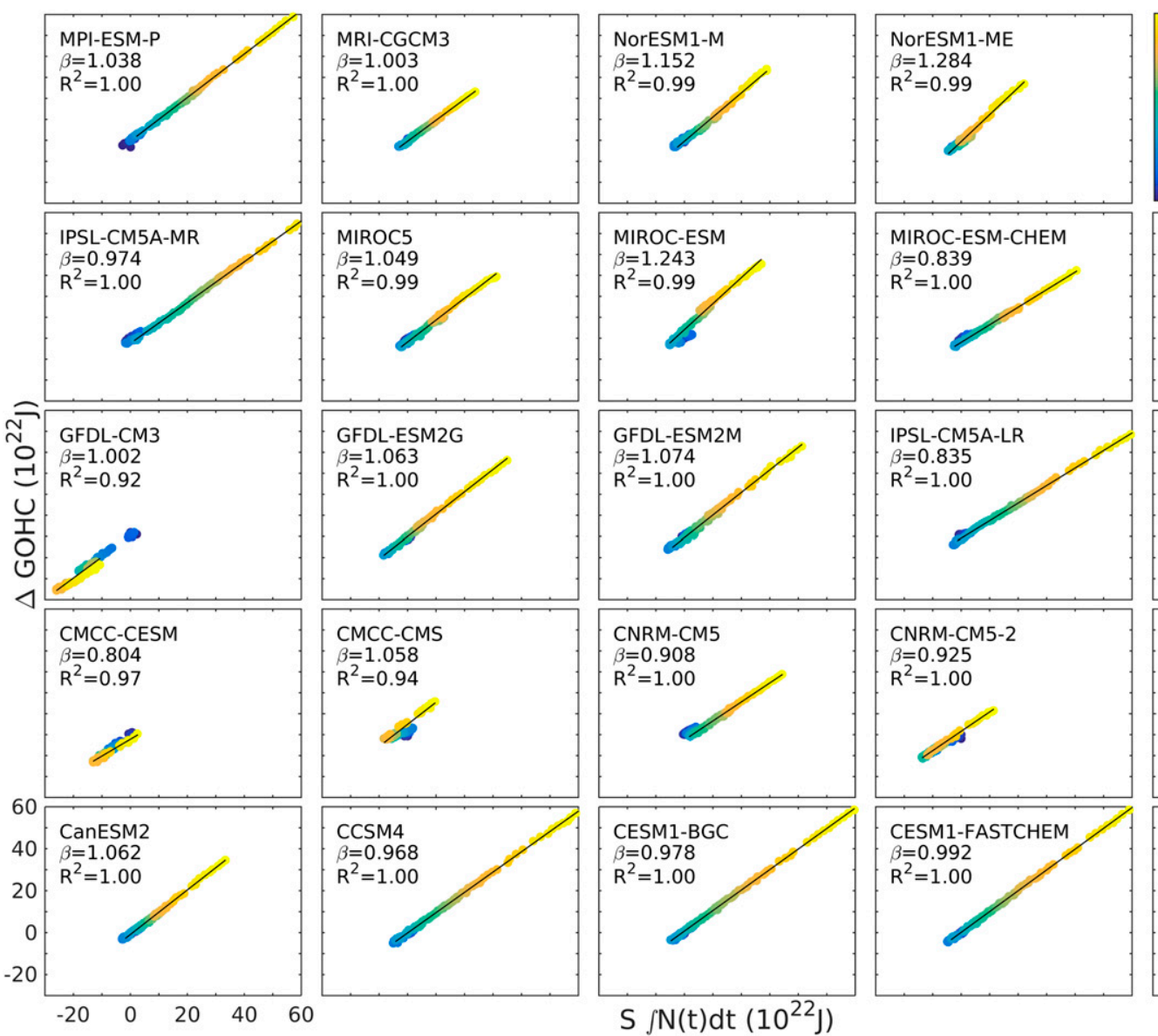
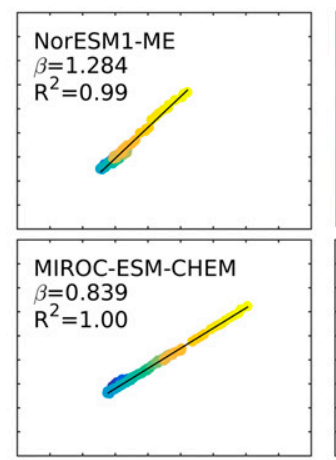

2000

1950

1900

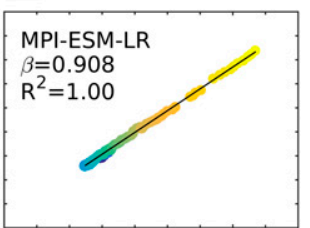

IPSL-CM5B-LR

$\beta=0.835$

$R^{2}=1.00$
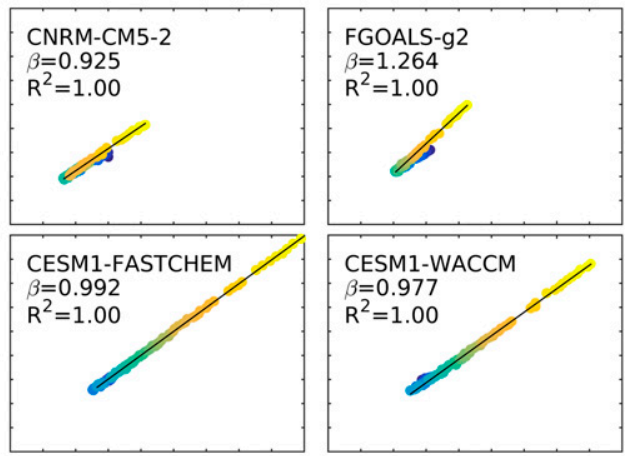

FIG. 4. Calculation of $\beta$ during the twentieth century. Each panel shows a scatterplot of global ocean heat uptake (relative to year 1900; $10^{22} \mathrm{~J}$ ) against global mean time-integrated net TOA flux $N$ multiplied by Earth's surface area $S\left(10^{22} \mathrm{~J}\right)$ for each CMIP5 climate model. Values of $\beta$ and of the coefficient of determination $R^{2}$ for the linear regression over 1900-2005 (black line) are indicated at the top left of each panel. Each panel has the same axes, shown by the bottom-left panel, and color bar (indicating years).

$$
\epsilon=\Delta \mathrm{GMTSL} / \mathrm{GOHU}
$$

We calculate $\epsilon$ by OLS regression of $\Delta$ GMTSL against GOHU using results from all available twentieth- and twenty-first-century scenarios (Figs. 6 and 7). The value of $\triangle$ GMTSL is computed as the anomaly in GMTSL relative to year 1866 for historical simulations and to year 2006 for projections. As stated above, GMTSL is corrected for model drift by removing a quadratic fit of GMTSL over the piControl segment parallel to the period considered (historical or projections). It is consistent with the model drift correction of $N$ and GOHU.

As for $\beta$, the linearity assumption (3) holds very well; the correlation coefficient between $\triangle$ GMTSL and GOHU exceeds 0.95 for all models and for each simulation (Figs. 6 and 7). However, $\epsilon$ varies across models because the thermal expansivity of seawater increases with temperature and pressure (Kuhlbrodt and Gregory 2012). Therefore, as for $\beta, \epsilon$ depends on the ocean heat pattern of the model. Since the heat pattern of a given model does not depend on the scenario at first order (Kuhlbrodt and Gregory 2012), $\epsilon$ does not depend on the scenario either. As for $\beta$, time variations of $\epsilon$ are very small $(<5 \%)$ and $\epsilon$ can be considered as constant with time over 1900-2100 at first order.

Our estimates of $\epsilon$ are in the range $0.12 \pm 0.02 \mathrm{~m} \mathrm{YJ}^{-1}$ $\left(1 \mathrm{YJ}=10^{24} \mathrm{~J}\right)$ in CMIP5 models (Table 1 ; when models with $\beta$ differing more than $20 \%$ from 0.93 were excluded). This is in agreement with the observational estimates of Levitus et al. (2005) and of Church et al. (2011), from which we infer $\epsilon=0.12 \pm 0.01 \mathrm{~m} \mathrm{YJ}^{-1}$ and $\epsilon=0.15 \pm 0.03 \mathrm{~m} \mathrm{YJ}^{-1}$, respectively. Our estimates of $\beta \epsilon$ are in agreement with the estimate of $\epsilon$ by Kuhlbrodt and Gregory (2012) [where $\epsilon$ was computed assuming $\beta=1$, as GOHU was approximated by the time integral (2)]. As $\epsilon$ was computed from a $1 \% \mathrm{yr}^{-1}$ increase in $\mathrm{CO}_{2}$ in Kuhlbrodt and Gregory (2012), the agreement with our values of $\beta \epsilon$ tends to be better for RCP scenarios 

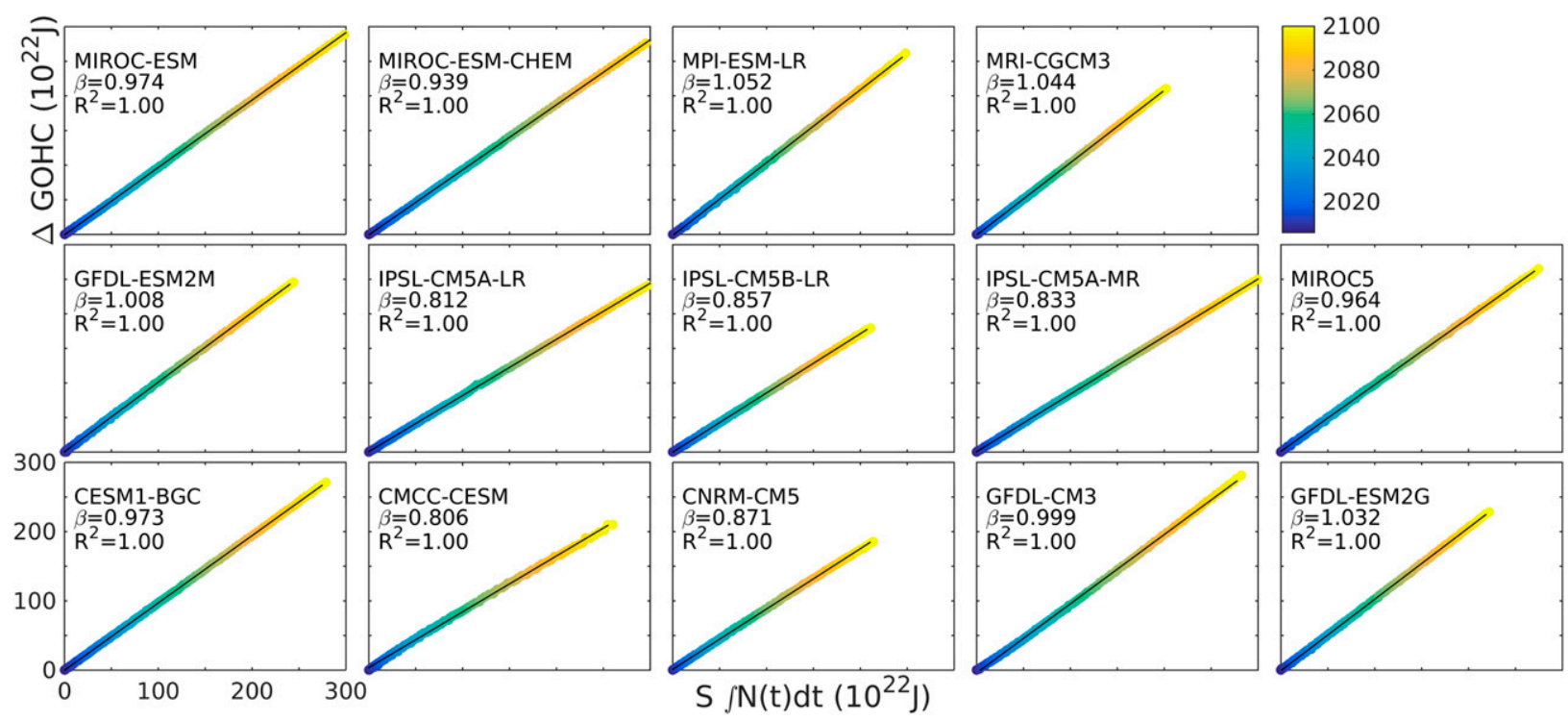

FIG. 5. Calculation of $\beta$ during the twenty-first century under the RCP8.5 scenario. Each panel shows a scatterplot of global ocean heat uptake (relative to year 2006; $10^{22} \mathrm{~J}$ ) against global mean time-integrated net TOA flux $N$ multiplied by Earth's surface area $S$ (in $10^{22} \mathrm{~J}$ ) for each CMIP5 climate model. Values of $\beta$ and of the coefficient of determination $R^{2}$ for the linear regression over 2006-99 (black line) are indicated at the top left of each panel. Each panel has the same axes, shown by the bottom-left panel, and color bar (indicating years).

than for the historical runs [see Table 1; auxiliary material of Kuhlbrodt and Gregory (2012)].

Potential correlations between $\beta$ and $\epsilon$ across CMIP5 models were tested using estimates from twentieth-century simulations (because more models are providing twentieth-century simulations than twenty-first-century simulations). We did not find any significant correlation between $\beta$ and $\epsilon$ across models, even though a correlation (even weak) could be possible since they both depend on the heat pattern of models.

\section{c. Sea level rise in response to the TOA radiative imbalance}

The heat accumulated in the ocean in response to the TOA radiative imbalance causes a rise in thermosteric sea level. Therefore, the rise in thermosteric sea level can be directly linked to the TOA radiative imbalance by the following equation:

$$
\Delta_{t_{\mathrm{ref}}, t} \mathrm{GMTSL}=S \beta \epsilon \int_{t_{\mathrm{ref}}}^{t} N(\tau) d \tau .
$$

We check (4) in climate models by plotting the regressions of the global thermosteric sea level rise $\Delta$ GMTSL against the right-hand side of (4) for all available twentieth- and twenty-first-century scenarios using the values of $\beta$ and $\epsilon$ computed in sections $3 \mathrm{a}$ and $3 \mathrm{~b}$. We find that in general $\int N d t$ provides a very strong constraint on $\triangle$ GMTSL (Figs. 8 and 9), which is consistent with the ocean storing most of the earth energy excess and global thermal expansion increasing linearly with global warming of the ocean (Figs. 4-7). The correlation coefficients range from 0.92 to 1.00 for twentieth-century simulations (Fig. 8, with the exception of CMCC-CMS) and equal 1.00 for twenty-first-century simulations (Fig. 9).

Across twentieth-century simulations, global thermosteric sea level rise over 1900-2005 ranges from +0 to $+76 \mathrm{~mm}$ (Figs. 2 and 10). Based on (4), we can compute the fraction of the across-model variance of $\Delta$ GMTSL explained by $\epsilon, \beta$, and $\int N d t$ (see appendixes $\mathrm{A}$ and $\mathrm{B})$. We $\mathrm{x}$ the across-model variance of $\epsilon, \beta$, and $\int N d t$ explains, respectively, $2 \%, 5 \%$, and $110 \%$ of the across-model variance of $\triangle$ GMTSL in twentiethcentury simulations. Note that the sum of acrossmodel variance explained by $\epsilon, \beta$, and $\int N d t$ exceeds $100 \%$. This is because there is a covariance between $\epsilon$ and $\int N d t$ that explains $-12 \%$ of the across-model variance of $\Delta$ GMTSL. This covariance is not significant (at the $90 \%$ confidence level) and arises by chance because of the finite size of our ensemble of models that provide twentieth-century simulations. For this reason the decomposition of variance must be regarded as approximate. However, given the very high level of variance explained by $\int N d t$, the decomposition of variance clearly shows, as expected, that the spread in models' TOA radiative imbalance explains most of the spread in $\Delta$ GMTSL over the twentieth century while the spreads in $\epsilon$ and $\beta$ play a minor role. 
TABLE 1. Fraction of the earth climate system energy excess associated with climate change stored in the ocean $\beta$, expansion efficiency of heat $\epsilon\left(10^{-25} \mathrm{~m} \mathrm{~J}^{-1}\right)$, transient thermosteric sea level response of the climate system $\mu\left(10^{-25} \mathrm{~m} \mathrm{~J}^{-1}\right)$, values of $\epsilon \beta \kappa /(\kappa+\beta \alpha)$ $\left(10^{-25} \mathrm{~m} \mathrm{~J}^{-1}\right.$, where $\kappa$ is the ocean heat uptake efficiency and $\alpha$ is the climate feedback parameter) for CMIP5 models for the historical 1900-2005 period (hist), RCP4.5, and RCP8.5 projections (for the period 2006-99). An asterisk after the model names indicates that volcanic forcing was prescribed during the piControl experiment of the model. The last two rows show the model ensemble mean and standard deviation (rms) of the different coefficients, including all available models (regardless of their value for $\beta$ ). (Expansions of model acronyms are available at http://www.ametsoc.org/PubsAcronymList.)

\begin{tabular}{|c|c|c|c|c|c|c|c|c|c|c|c|c|}
\hline \multirow[b]{2}{*}{ Model } & \multicolumn{3}{|c|}{$\beta$} & \multicolumn{3}{|c|}{$\epsilon$} & \multicolumn{3}{|c|}{$\mu$} & \multicolumn{3}{|c|}{$\epsilon \beta \kappa /(\kappa+\beta \alpha)$} \\
\hline & Hist & $\mathrm{RCP} 4.5$ & RCP8.5 & Hist & $\mathrm{RCP} 4.5$ & RCP8.5 & Hist & $\mathrm{RCP} 4.5$ & RCP8.5 & Hist & $\mathrm{RCP} 4.5$ & RCP8.5 \\
\hline ACCESS1.0 & - & - & - & 1.024 & - & - & - & - & - & - & - & - \\
\hline ACCESS1.3 & - & - & - & 1.097 & - & - & - & - & - & - & - & - \\
\hline CanESM2 & 1.062 & - & - & 1.218 & - & - & 0.365 & - & - & 0.400 & - & - \\
\hline CCSM4 & 0.968 & - & - & 1.149 & - & - & - & - & - & - & - & - \\
\hline CESM1(BGC) & 0.978 & 0.978 & 0.973 & 1.136 & 1.094 & 1.167 & - & - & - & - & - & - \\
\hline CESM1(CAM5) & - & - & - & 1.311 & - & - & - & - & - & - & - & - \\
\hline CESM1(FASTCHEM) & 0.992 & - & - & 1.149 & - & - & - & - & - & - & - & - \\
\hline CESM1(WACCM) & 0.977 & - & - & 1.150 & - & - & - & - & - & - & - & - \\
\hline CMCC-CESM & 0.804 & - & 0.806 & 1.247 & - & 1.234 & - & - & - & - & - & - \\
\hline CMCC-CMS & 1.058 & 1.088 & - & 1.333 & 1.208 & - & - & - & - & - & - & - \\
\hline CNRM-CM5* & 0.908 & 0.862 & 0.871 & 1.249 & 1.202 & 1.249 & 0.480 & 0.508 & 0.496 & 0.351 & 0.332 & 0.346 \\
\hline CNRM-CM5.2* & 0.925 & - & - & 1.277 & - & - & - & - & - & - & - & - \\
\hline CSIRO Mk3.6.0 & - & - & - & 1.060 & - & - & - & - & - & - & - & - \\
\hline FGOALS-g2* & 1.264 & - & - & 1.358 & - & - & - & - & - & - & - & - \\
\hline FIO-ESM & - & - & - & - & - & - & - & - & - & - & - & - \\
\hline GFDL CM3 & 1.002 & 1.000 & 0.999 & 1.321 & 1.222 & 1.255 & - & 0.674 & 0.710 & - & 0.572 & 0.587 \\
\hline GFDL-ESM2G & 1.063 & - & 1.032 & 1.120 & - & 1.103 & 0.535 & - & 0.476 & 0.432 & - & 0.364 \\
\hline GFDL-ESM2M & 1.074 & 1.018 & 1.008 & 1.217 & 1.164 & 1.206 & 0.691 & 0.619 & 0.564 & 0.385 & 0.395 & 0.408 \\
\hline GISS-E2-H-CC* & - & - & - & 1.171 & - & - & - & - & - & - & - & - \\
\hline GISS-E2-R-CC* & - & - & - & 1.097 & - & - & - & - & - & - & - & - \\
\hline GISS-E2-R* & - & - & - & 1.093 & - & - & - & - & - & - & - & - \\
\hline HadGEM2-ES* & - & - & - & 1.070 & - & - & - & - & - & - & - & - \\
\hline IPSL-CM5A-LR* & 0.835 & - & 0.812 & 1.222 & - & 1.210 & 0.539 & - & 0.531 & 0.540 & - & 0.474 \\
\hline IPSL-CM5B-LR* & 0.834 & 0.845 & 0.857 & 1.290 & 1.232 & 1.279 & - & - & - & - & - & - \\
\hline IPSL-CM5A-MR* & 0.974 & 0.794 & 0.833 & 1.089 & 1.163 & 1.231 & 0.687 & 0.504 & 0.551 & 0.510 & 0.441 & 0.477 \\
\hline MIROC5* & 1.049 & 0.984 & 0.964 & 1.194 & 1.185 & 1.212 & 0.348 & - & - & 0.338 & - & - \\
\hline MIROC-ESM* & 1.243 & 0.996 & 0.974 & 1.142 & 1.205 & 1.252 & - & - & - & - & - & - \\
\hline MIROC-ESM-CHEM* & 0.839 & 0.944 & 0.939 & 1.192 & 1.202 & 1.252 & - & - & - & - & - & - \\
\hline MPI-ESM-LR & 0.908 & - & 1.052 & 1.198 & - & 1.199 & 0.488 & - & 0.551 & 0.372 & - & 0.404 \\
\hline MPI-ESM-P & 1.039 & - & - & 1.179 & - & - & - & - & - & - & - & - \\
\hline MRI-CGCM3 & 1.003 & 1.064 & 1.044 & 1.154 & 1.209 & 1.234 & 0.390 & 0.501 & 0.492 & 0.335 & 0.346 & 0.351 \\
\hline NorESM1-M* & 1.152 & - & - & 1.077 & - & - & - & - & - & - & - & - \\
\hline NorESM1-ME* & 1.284 & - & - & 1.217 & - & - & - & - & - & - & - & - \\
\hline Model mean & 1.008 & 0.961 & 0.940 & 1.141 & 1.190 & 1.220 & 0.564 & 0.561 & 0.546 & 0.396 & 0.417 & 0.426 \\
\hline Model rms & 0.134 & 0.092 & 0.088 & 0.223 & 0.038 & 0.044 & 0.148 & 0.081 & 0.074 & 0.050 & 0.096 & 0.082 \\
\hline
\end{tabular}

For the twenty-first-century simulations under the RCP8.5 scenario, the size of the ensemble of models providing simulations is smaller than for twentiethcentury simulations (Table 1). The decomposition of variance is thus even more approximate than for twentieth-century simulations. Still, the variance in TOA radiative imbalance explains most (about 95\%) of the variance in $\triangle$ GMTSL (Fig. 11).

\section{d. Sea level rise in response to the radiative forcing of the climate system under transient climate}

The global energy budget of Earth indicates that the TOA radiative imbalance $N$ is the sum of the radiative forcing of the climate system $F$ and the radiative response of the climate system, which is proportional to the globally averaged surface temperature change $\Delta T$ :

$$
N=F-\alpha \Delta T,
$$

where $\alpha$ is the climate feedback parameter (watts per meter squared per kelvin; Gregory et al. 2004).

Gregory and Forster (2008) showed that in transient climate, under continuously increasing forcing, the global surface temperature change $\Delta T$ linearly depends on the radiative forcing $F$ so that $F=\rho \Delta T$, where $\rho$ is the climate resistance (watts per meter 


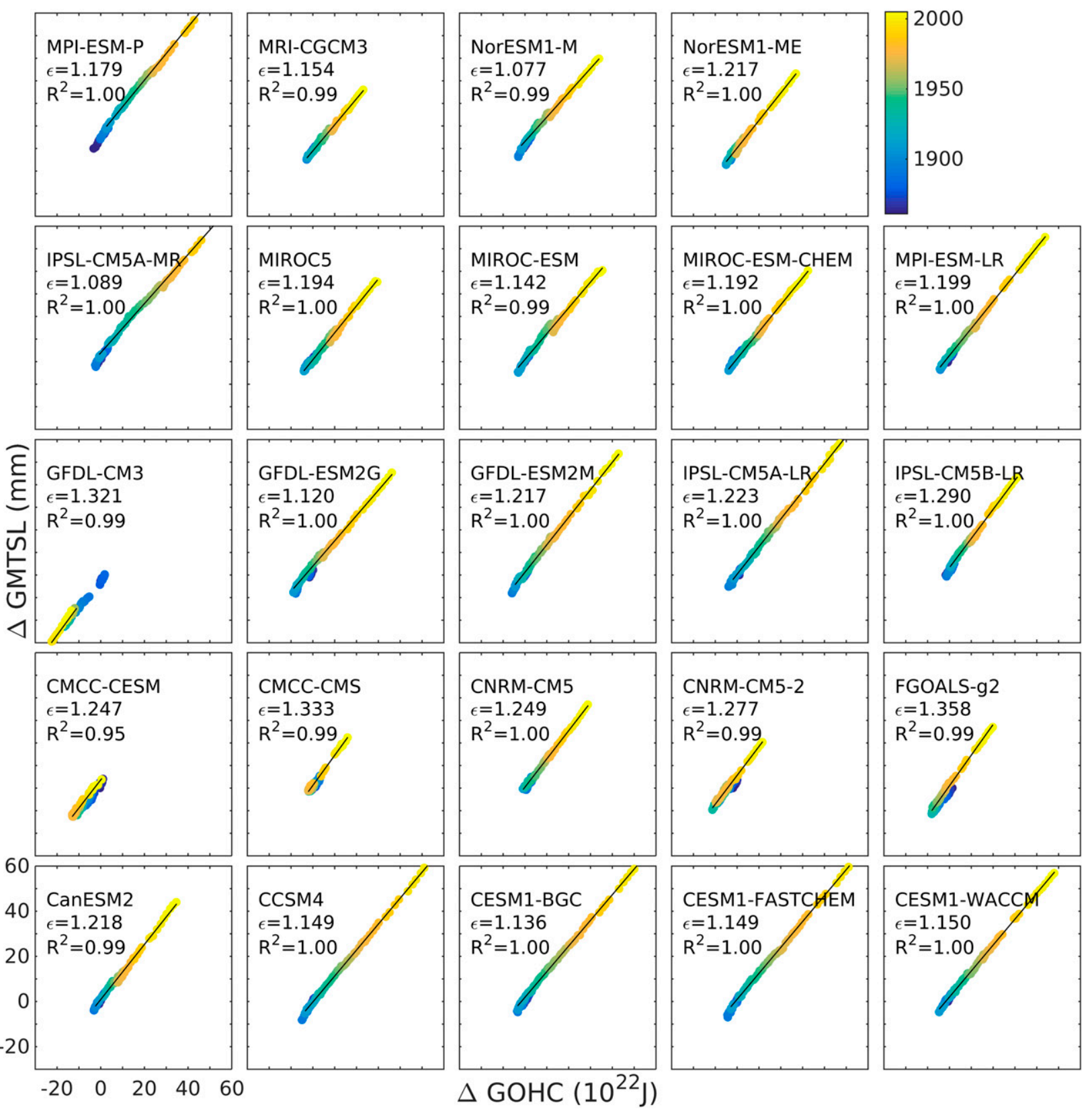

FIG. 6. Calculation of $\epsilon$ during the twentieth century. Each panel shows a scatterplot of global mean thermosteric sea level rise (relative to year 1900; mm) against ocean heat uptake (relative to year 1900; $10^{22} \mathrm{~J}$ ) for each CMIP5 climate model. Values of $\epsilon$ and of the coefficient of determination $R^{2}$ for the linear regression over 1900-2005 (black line) are indicated at the top left of each panel. Each panel has the same axes, shown by the bottom-left panel, and color bar (indicating years).

squared per kelvin). The climate resistance characterizes the radiative forcing needed to increase the global mean surface temperature by $1 \mathrm{~K}$. It actually depends on the climate feedback parameter and the ocean heat uptake efficiency $\kappa$ through the relation given by $\rho=\alpha+\kappa / \beta$ (see appendix A). The linear relationship $F=\rho \Delta T$ has no time dependence, implying that under continuously increasing forcing, global surface temperature changes track radiative forcing changes. As a direct consequence, TOA imbalance also tracks radiative forcing changes since $N$ verifies the following: $N=F-\alpha \Delta T=[(\rho-\alpha) / \rho] F=$ $[\kappa /(\kappa+\beta \alpha)] F$. Sea level rise linearly depends on the time-integrated TOA radiative imbalance (see section 3c). Therefore, under continuously increasing forcing, it should also linearly depend on the time-integrated 


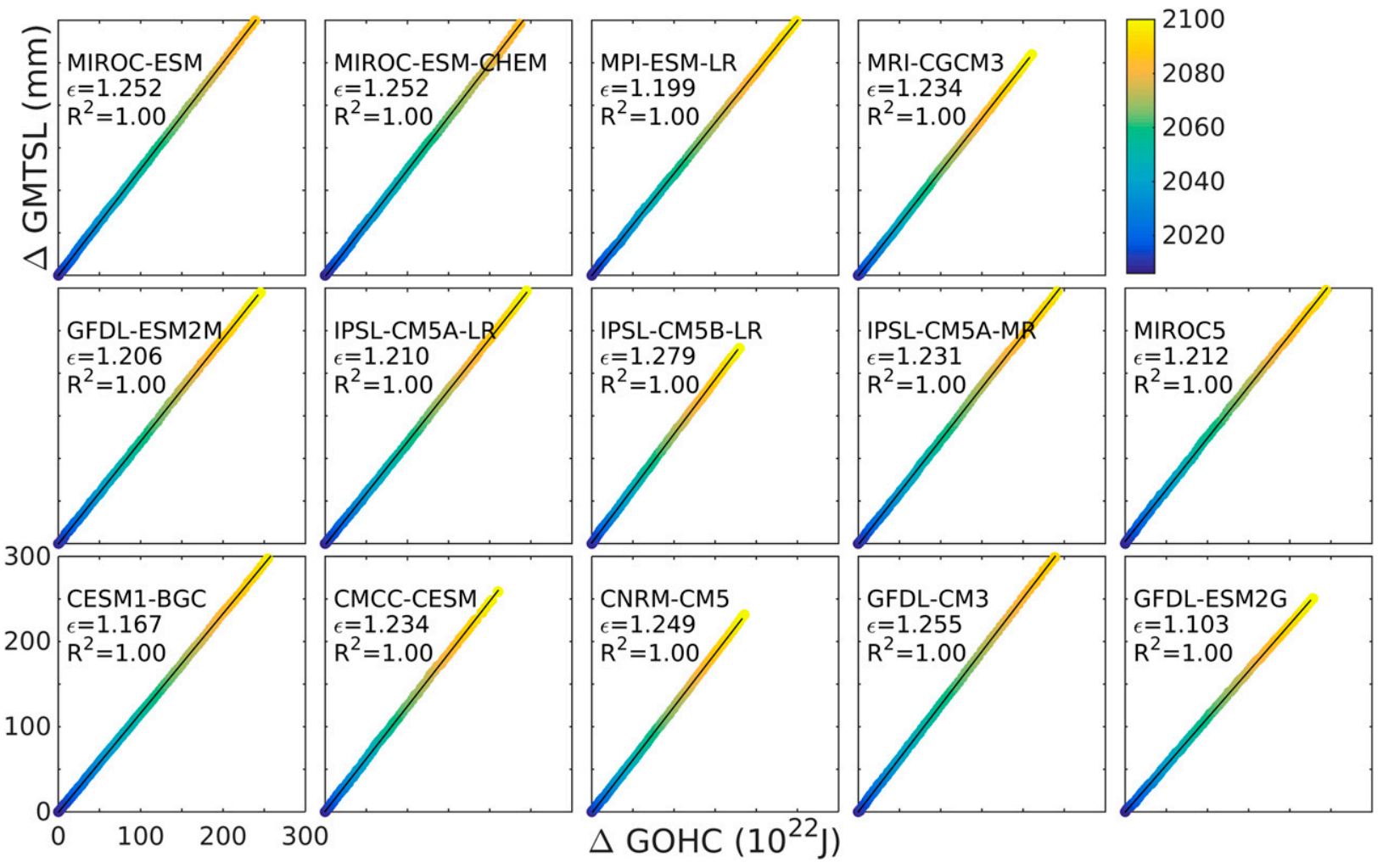

FIG. 7. Calculation of $\epsilon$ during the twenty-first century under the RCP8.5 scenario. Each panel shows a scatterplot of global mean thermosteric sea level rise (relative to year 2006; mm) against ocean heat uptake (relative to year 2006; $10^{22} \mathrm{~J}$ ) for each CMIP5 climate model. Values of $\epsilon$ and of the coefficient of determination $R^{2}$ for the linear regression over 2006-99 (black line) are indicated at the top left of each panel. Each panel has the same axes, shown by the bottom-left panel, and color bar (indicating years).

radiative forcing of the climate system following this equation:

$$
\Delta_{t_{\mathrm{ref}}, t} \mathrm{GMTSL}=S \beta \epsilon \frac{\kappa}{\kappa+\beta \alpha} \int_{t_{\mathrm{ref}}}^{t} F(\tau) d \tau
$$

Equation (6) is consistent with the results of Bouttes et al. (2013), who noted that the global mean thermal expansion of the ocean linearly depends on the time-integrated forcing. In short, (6) implies a linear relationship between GMTSL variations and the time-integrated radiative forcing $\int F d \tau$, as in the following equation:

$$
\Delta \mathrm{GMTSL}=\mu S \int_{t_{\mathrm{ref}}}^{t} F(\tau) d \tau
$$

where $\mu$ is a constant with units of meters per joule. This linear relationship is expected to hold only under transient climate change when the forcing is steadily increasing. It is the analog for sea level of the relationship $\Delta T=F / \rho$ between the global mean surface air temperature $\Delta T$, the radiative forcing $F$, and the climate resistance $\rho$. For this reason we call $\mu$ the transient thermosteric sea level response of the climate system.

Observations and climate model simulations of the twentieth century show that the linear relationship $F=\rho \Delta T$ holds under the present-day climate change (Gregory and Forster 2008). They also indicate that it holds over the twenty-first century but only for scenarios with steadily increasing forcing (i.e., for scenarios RCP4.5, RCP6.0, and RCP8.5; Gregory and Forster 2008). Consequently, the linear relation between $\triangle$ GMTSL and the time-integrated radiative forcing of the climate system based on (6), which is based on the assumption that $F=\rho \Delta T$, should also hold in twentiethand twenty-first-century simulations (except for the scenario RCP2.6). We now check the validity of this assumption with CMIP5 climate model simulations. Following Forster et al. (2013), we calculate $F$ as the sum of the TOA imbalance $N$ and the climate radiative response $\alpha \Delta T$ [see (5)]. The values of $\alpha$ were taken from the estimates of Kuhlbrodt and Gregory (2012) under the $1 \% \mathrm{yr}^{-1}$ increase of $\mathrm{CO}_{2}$ scenario. The globally averaged surface temperature changes are detrended by removing the linear regression of $\Delta T$ over the piControl 


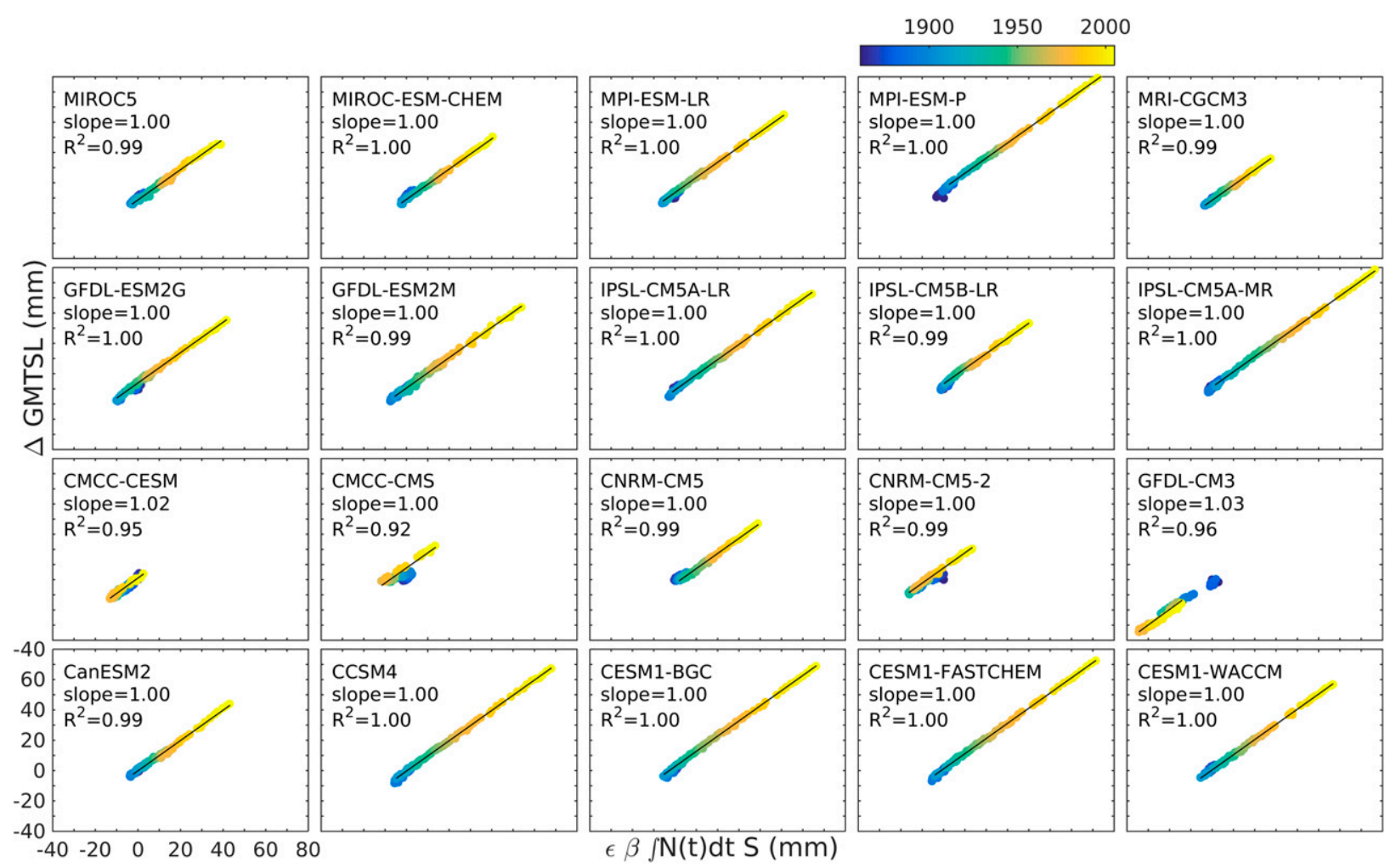

FIG. 8. Scatterplots of global mean thermosteric sea level rise relative to year $1900 \Delta$ GMTSL (mm) against values of $S \epsilon \beta \int_{1900} N(t) d t$ $(\mathrm{mm})$ during the twentieth century for each available model. Values of the slope and of the coefficient of determination $R^{2}$ for the linear regression over 1900-2005 (black line) are indicated at the top left of each panel. Each panel has the same axes, shown by the bottom-left panel, and color bar (indicating years).

segment parallel to the period considered (historical or projection). The variable $F$ is calculated with annual times series of $N$ and $\Delta T$. We then regress $\Delta$ GMTSL against $S \int F d t$ for twentieth- and twenty-first-century simulations to check the validity of relation (7).

Scatterplots in Figs. 12 and 13 show that the linear relation between $\triangle$ GMTSL and the integrated radiative forcing of the climate system $S \int F d t$ holds very well for all models under the twentieth and twenty-first centuries. The linear relationship is not perfect because of unforced variability generated internally on interannual to multidecadal time scales by the climate system. Nevertheless, the correlation coefficients are very high $(>0.95)$.

Deviations from the linear relationship occur in twentieth-century simulations during years affected by volcanic forcing because during these years, $\Delta$ GMTSL responds more weakly to changes in $F$ than it does over the rest of the twentieth century in response to the long term change in $F$. This feature suggests that the response of the ocean (in terms of heat content) to the forcing resulting from volcanic eruptions is different from its response to the long-term anthropogenic forcing. More investigation is needed to determine whether this is due to the different nature of the volcanic forcing (emission of sulfate aerosols and associated radiative-dynamical response of the stratosphere and the troposphere) or to its episodic and short-duration occurrences.

Deviations from the linear relationship between $\Delta$ GMTSL and $\int F d t$ also become apparent in twentyfirst-century simulations in which no volcanic eruptions are prescribed. For the same increase in $\int F d t, \Delta$ GMTSL tends to increase more slowly toward the end of the twenty-first century (i.e., $\mu$ tends to decrease toward the end of the twenty-first century), when the forcing continues to rise. This deceleration in $\triangle$ GMTSL as the forcing increases is simulated by all models in Fig. 13. If in the real world $\mu$ tends to decrease similarly as $F$ increases, $\triangle$ GMTSL projections based on (7) will tend to slightly overestimate the real GMTSL rise, and the overestimation will get more severe for projections as the forcing rises. Note that these time variations of $\mu$ are small $(<10 \%)$, and, at a first order, $\mu$ can be considered as constant over the range of forcing covered by historical and twenty-first-century simulations (Table 1). 


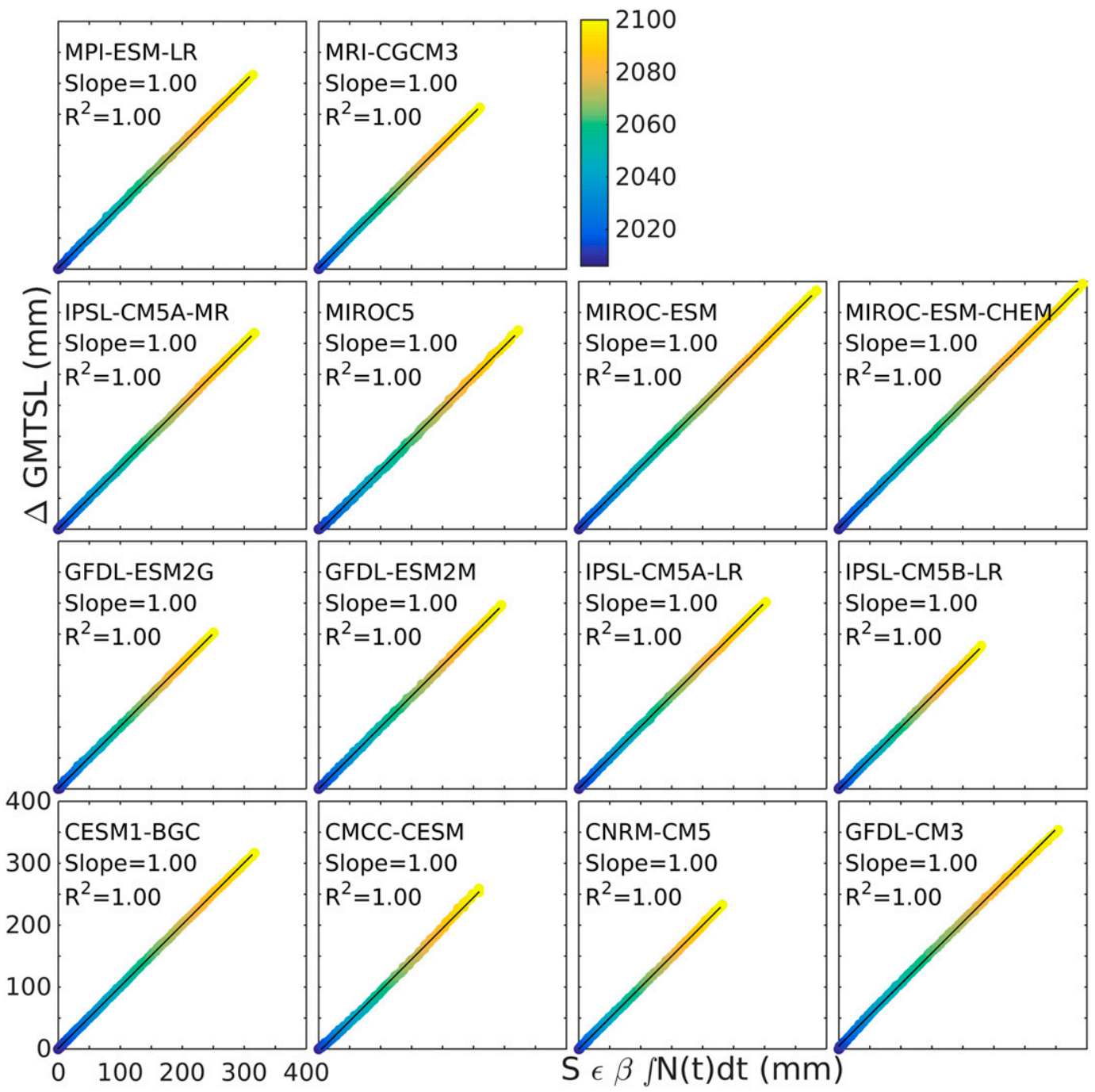

FIG. 9. Scatterplots of global mean thermosteric sea level rise relative to year $2006 \Delta$ GMTSL (in mm) against values of $S \epsilon \beta \int_{2006} N(t) d t(\mathrm{~mm})$ during the twenty-first century under the RCP8.5 scenario for each available model. Values of the slope and of the coefficient of determination $R^{2}$ for the linear regression over 2006-99 (black line) are indicated at the top left of each panel. Each panel has the same axes, shown by the bottom-left panel, and color bar (indicating years).

\section{e. Origins of the spread in GTMSL rise across climate models}

In summary, climate models indicate that the linearity assumption between $\Delta$ GMTSL and $S \int F d t$ (7) holds very well in current climate change. For any given climate model, the annual time series of the global thermosteric mean sea level rise $\Delta$ GMTSL in twentieth- and twenty-first-century simulations can be deduced with very good accuracy (except during the few years following explosive volcanic eruptions) from the radiative forcing with (7). We use the linear relation (7) to understand the causes of the spread in $\triangle$ GMTSL rise across climate models. With this simple relation, we can compute the across-model variance in global thermosteric mean sea level rise explained by the acrossmodel variance in $F$ and $\mu$ (see appendix B). We check for any potential covariances between $\mu$ and $\int F(t) d t$ before the computation. For the ensemble of historical simulations, the time-integrated forcing explains $24 \%$ of the across-model variance in $\Delta$ GMTSL while $\mu$ explains $73 \%$. Results are different for the ensemble of RCP8.5 simulations. The time-integrated forcing explains only $25 \%$ of the variance while $\mu$ explains more than $90 \%$ (similar results are found for the ensemble of RCP4.5 simulations; see supplementary material). In addition, a small and significant covariance arises between $\mu$ and $\int F(t) d t$ because of the small size of the 


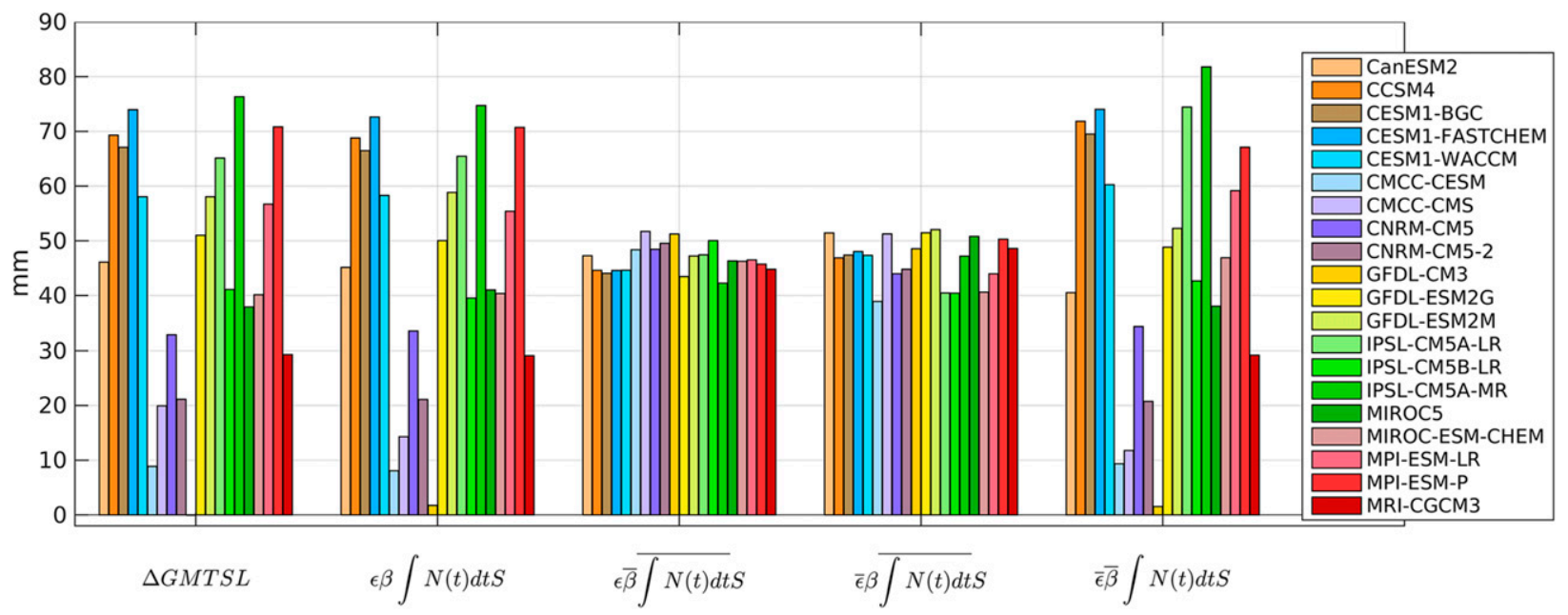

FIG. 10. Climate model global mean thermosteric sea level rise in 2005 relative to $1900(\mathrm{~mm})$ computed from the 3D temperature and salinity fields (first group), from the climate coefficient relationship $\epsilon \beta \int N(t) d t S$ (second group), from the climate coefficient relationship using the model ensemble mean values for $\beta$ and $\int N(t) d t$ (third group), for $\epsilon$ and $\int N(t) d t$ (fourth group), and for $\epsilon$ and $\beta$ (fifth group). The overbar denotes an averaging across the model ensemble. Only models conserving energy ( $\beta$ within $20 \%$ of 0.93 ) are included.

RCP8.5 model ensemble. This covariance explains less than $15 \%$ of the variance of $\triangle$ GMTSL, and we neglect it.

The fraction of $\triangle$ GMTSL variance explained by $F$ is larger in historical simulations than in projections because the across-model spread in $F$ is larger in historical simulations. The reason is that the aerosol forcing, which is highly uncertain and very different across models, is relatively important compared to the total radiative forcing over the twentieth century. Therefore, aerosol forcing tends to generate a large across-model spread in radiative forcing $F$ over the twentieth century. In contrast, over the twenty-first century, the GHG forcing increases and aerosol forcing is projected to decrease. As a consequence, the spread in total radiative forcing $F$ is reduced over the twentyfirst century.

The fraction of $\Delta$ GMTSL variance explained by $\mu$ is large in both historical and twenty-first-century simulations because the across -model variance in $\mu$ is large in these simulations (see Figs. 14 and 15 ; Table 1). Indeed, $\mu$ depends on the climate feedback parameter $\alpha$ and on the ocean heat uptake efficiency $\kappa$, which both vary widely across models (Gregory and Forster 2008; Kuhlbrodt

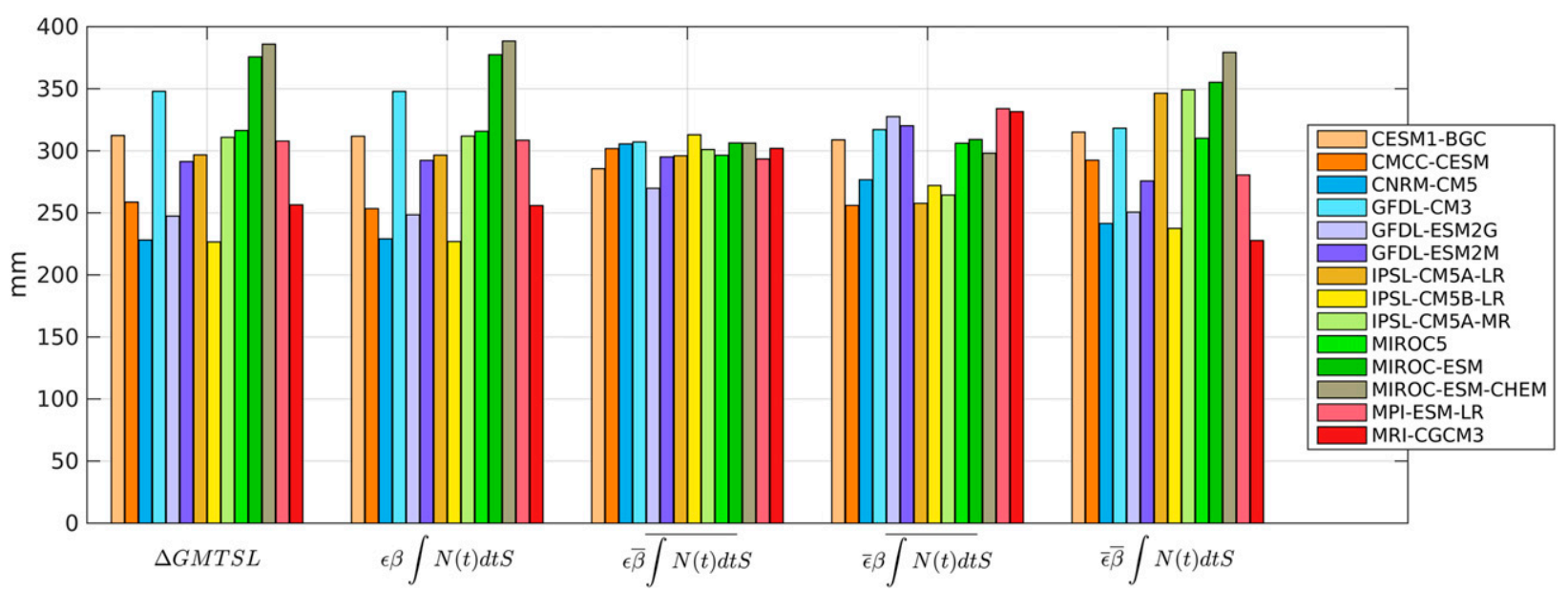

FIG. 11. Climate model global mean thermosteric sea level rise in 2099 relative to $2006(\mathrm{~mm})$ under the RCP8.5 scenario computed from the $3 \mathrm{D}$ temperature and salinity fields (first group), from the climate coefficient relationship $\epsilon \beta \int N(t) d t S$ (second group), from the climate coefficient relationship using the model ensemble mean values for $\beta$ and $\int N(t) d t$ (third group), for $\epsilon$ and $\int N(t) d t$ (fourth group), and for $\epsilon$ and $\beta$ (fifth group). The overbar denotes an averaging across the model ensemble. Only models conserving energy ( $\beta$ within $20 \%$ of 0.93$)$ are included. 


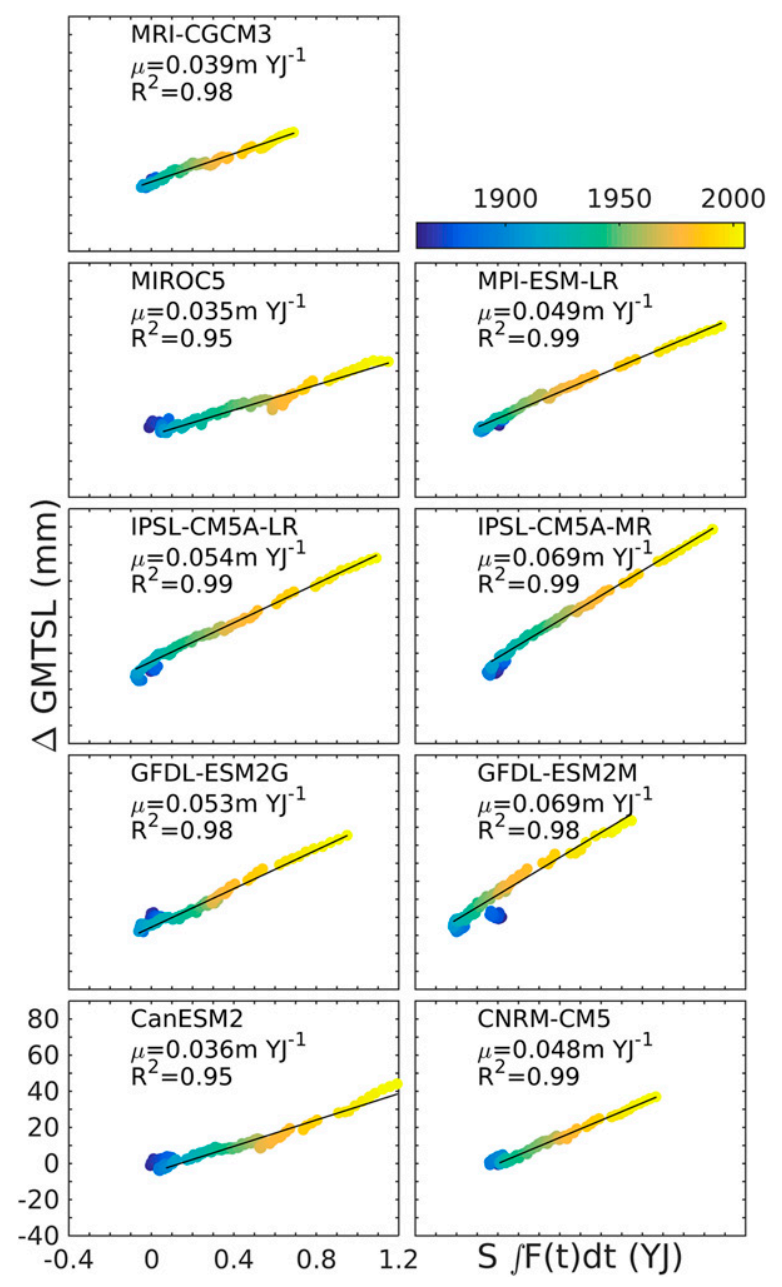

FIG. 12. Scatterplots of global mean thermosteric sea level rise relative to year $1900 \Delta$ GMTSL (mm) against values of $S \int_{1900} F(t) d t$ $\left(10^{24} \mathrm{~J}\right)$ during the twentieth century for each available model. Values of the slope $\mu$ and of the coefficient of determination $R^{2}$ for the linear regression over 1900-2005 (black line) are indicated at the top left of each panel. Each panel has the same axes, shown by the bottom-left panel, and color bar (indicating years).

and Gregory 2012). According to (6), under continuously increasing forcing $\mu$ can be approximated by the following:

$$
\mu=\frac{\epsilon \beta \kappa}{\kappa+\beta \alpha}
$$

We check to what extent this approximation is verified in twentieth- and twenty-first-century simulations by comparing our estimates of $\mu$ to estimates based on published estimates of $\alpha$ and $\kappa$. Several past studies have calculated $\alpha$ and $\kappa$ for CMIP5 climate models and they show generally consistent results (Andrews et al. 2012; Kuhlbrodt and Gregory 2012; Forster et al. 2013). We chose to use Kuhlbrodt and Gregory (2012) estimates because they provide values of both $\alpha$ and $\kappa$ for several models considered in the present study under the $1 \% \mathrm{yr}^{-1} \mathrm{CO}_{2}$ increase, which is a scenario closer to historical and projected emissions than the abrupt doubling or quadrupling $\mathrm{CO}_{2}$ scenarios.

Figures 16 and 17 compares our calculation of $\mu$ against the estimate of $\epsilon \beta \kappa /(\kappa+\beta \alpha)$. The estimate based on Kuhlbrodt and Gregory (2012) is biased low (Table 1), but it tends to reproduce the across-model spread in $\mu$. This result shows that the relation between $\mu, \alpha$, and $\kappa$ is well captured by (8) (except for the historical simulations of the GFDL-ESM2G and IPSL-CM5-LR models; see Fig. 16), and this relation holds in historical and twenty-first-century climate model simulations. The estimate based on the values of Kuhlbrodt and Gregory (2012) for $\kappa$ and $\alpha$ is probably biased low because it is calculated with values of $\alpha$ and $\kappa$ accounting for the $\mathrm{CO}_{2}$ forcing alone. A calculation with all forcings, including the natural forcing (solar activity and volcanic forcing but excluding years impacted by explosive eruptions) and the aerosol forcing, would result in higher values of the climate resistance $\rho=\alpha+\kappa$ (Gregory and Forster 2008) and hence in lower values of $\mu$, which is more consistent with our estimates.

In the small ensemble of climate models for which we were able to compute the different climate coefficients, $\alpha$ and $\kappa$ are actually highly correlated. Therefore, we could neither statistically and robustly explain the across-model variance in $\triangle$ GMTSL due to the acrossmodel variance in the different climate coefficients nor compare our results to Kuhlbrodt and Gregory (2012). Nevertheless, the variance in $\alpha$ dominates the variance in $\Delta$ GMTSL. The variance in $\kappa$ explains a substantial, but less important, fraction of the variance in $\Delta$ GMTSL.

\section{Discussion and conclusions}

Climate model simulations of twentieth- and twenty-first-century (RCP4.5 and RCP8.5 scenarios) climate change indicate a linear relationship between the global mean thermosteric sea level (GMTSL) rise and the time-integrated radiative forcing $\int F d \tau$ following the equation $\Delta \mathrm{GMTSL}=\mu S \int F d \tau$, where $S$ is the surface area of Earth and $\mu$ is the transient thermosteric sea level response of the climate system. This linear relationship with the parameter $\mu$ holds only in transient climate change when the forcing is steadily increasing. Climate model simulations of the twentieth and twenty-first centuries indicate a range for $\mu$ of $0.055 \pm 0.025 \mathrm{~m} \mathrm{YJ}^{-1}$. This is in agreement with the observational estimates of Church et al. (2011) from which we infer that $\mu=0.05 \pm 0.01 \mathrm{~m} \mathrm{YJ}^{-1}$. Note that the uncertainty in observed $\mu$ is smaller than the uncertainty 


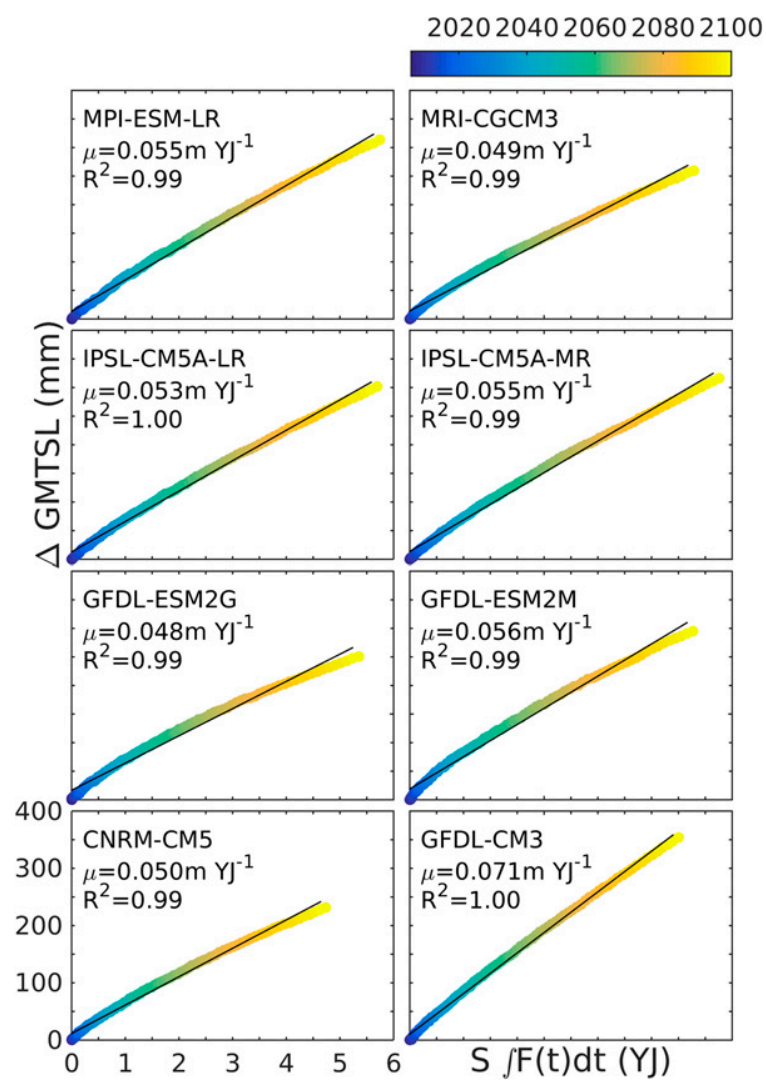

FIG. 13. Scatterplots of global mean thermosteric sea level rise relative to year $2006 \Delta$ GMTSL (mm) against values of $S \int_{2006} F(t) d t$ $\left(10^{24} \mathrm{~J}\right)$ during the twenty-first century for each available model under the RCP8.5 scenario. Values of the slope $\mu$ and of the coefficient of determination $R^{2}$ for the linear regression over 2006-99 (black line) are indicated at the top left of each panel. Each panel has the same axes, shown by the bottom-left panel, and color bar (indicating years).

in $\mu$ from climate models. This result suggests that observations could be used to constrain climate models and reduce their range in $\mu$. Yet, the uncertainty in $\mu$ from observations (Church et al. 2011) is artificially low because it does not include the uncertainty in volcanic forcing and anthropogenic aerosol forcing. If we include the uncertainty from anthropogenic aerosol forcing, which is particularly large, the range of observed $\mu$ becomes too large to give any constraint on climate models.

Differences in $\mu$ across CMIP5 climate models lead to differences in estimates of GMTSL rise over the twentieth and twenty-first centuries among climate models. The value of $\mu$ varies across models because it depends on the climate feedback parameter $\alpha$ and the ocean heat uptake efficiency $\kappa$, which both vary across models. The across-model spread in $\alpha$ essentially comes from differences in feedback processes (particularly, but not only, the cloud feedback) to the radiative forcing perturbation across models (Webb et al. 2006; Andrews et al. 2012). The spread in $\kappa$ is essentially related to across-model differences in heat transport processes within the ocean (e.g., Kuhlbrodt and Gregory 2012; Hallberg et al. 2013). The across-model spread in $\mu$, which is generated by these differences in atmospheric and oceanic processes among climate models, explains most of the acrossmodel spread in GMTSL rise over the twentieth and twenty-first centuries. Note that the spread in $\mu$ is not the only contributor to the across-model spread in GMTSL rise. Differences in radiative forcing $F$, which come from differences in prescribed natural and anthropogenic forcing (Collins et al. 2013), differences in the treatment of radiative transfer (Collins et al. 2006), and differences in the rapid tropospheric and land surface adjustment processes (Gregory and Webb 2008) contribute as well to the across-model GMTSL spread. Over the twenty-first century, $F$ tends to be more homogeneous across models because the anthropogenic aerosol forcing, which is one of the largest sources of uncertainty in $F$, is smaller compared to other forcings than over the twentieth century. For this reason, $F$ explains less variance in GMTSL rise across models in twenty-first-century simulations than in twentiethcentury simulations.

The results presented in this study for the global mean thermosteric sea level rise are not expected to hold at regional scales. Indeed, at regional scales, the spread in $\kappa$ is expected to explain a larger fraction of the thermosteric sea level rise. Improvements of parameterizations of small-scale processes that impact the vertical ocean heat transport, such as small and mesoscale eddies or turbulent mixing, are therefore required for improved skills of regional sea level simulation (e.g., Kuhlbrodt and Gregory 2012; Hallberg et al. 2013; Melet et al. 2015, manuscript submitted to J. Climate).

The comparison of the simulated global mean thermosteric sea level rise to observational estimates for the 0-700-m depth layer and over 1961-2005 (i.e., when the observations became nearly global) shows that only a few climate models reproduce the observed thermosteric sea level within error bars. Most models tend to overestimate the GMTSL rise over 1961-2005 either because their radiative forcing is too large or because their $\mu$ is too large, or a combination of both. Among the nine models for which we have been able to compute $\mu$, only three (namely CNRM-CM5, MIROC5, and MRI-CGCM3) agree within error bars with observations of the thermosteric sea level over 1961-2005. Interestingly, these three models tend to have a small $\mu$ (in the range $0.035-0.048 \mathrm{~m} \mathrm{YJ}^{-1}$ ). The six other models, which overestimate the thermosteric sea level rise, tend to have a larger $\mu$ (in the range 


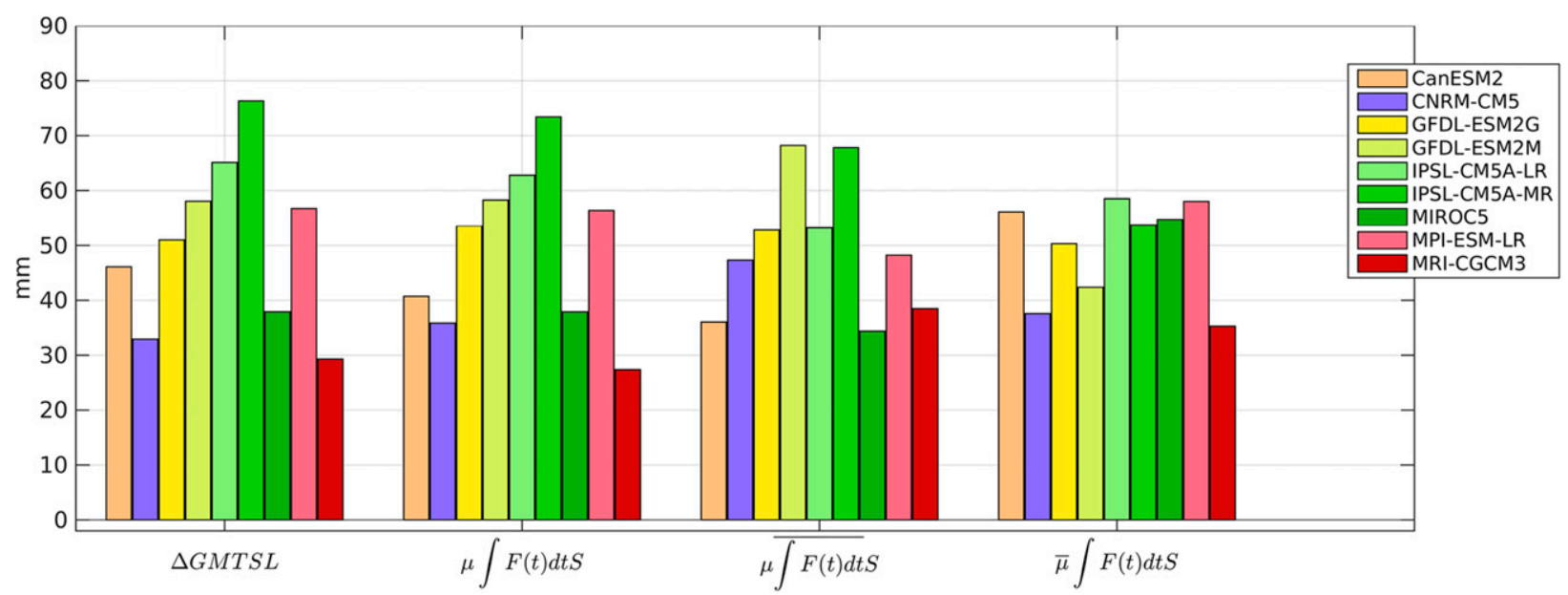

FIG. 14. Climate model global mean thermosteric sea level rise in 2005 relative to $1900(\mathrm{~mm})$ computed from the 3D temperature and salinity fields (first group), from the climate coefficient relationship $\mu \int F(t) d t S$ (second group), from the climate coefficient relationship using the model ensemble mean values for $\int F(t) d t$ (third group), and for $\mu$ (fourth group). The overbar denotes an averaging across the model ensemble. Only models conserving energy ( $\beta$ within $20 \%$ of 0.93 ) are included.

$0.037-0.069 \mathrm{~m} \mathrm{YJ}^{-1}$ ). This result suggests that models generally overestimate thermosteric sea level rise compared to observations because their $\mu$ is too large. If this result is confirmed with more climate models and if we can reduce the uncertainty on twentieth-century radiative forcing across models, thermosteric sea level change observations could give an interesting constraint on $\mu$ estimates from climate models. This would help reduce the across-model spread in $\mu$ and would lead to more accurate projections of future global mean thermosteric sea level rise.

The linear relationship between $\triangle$ GMTSL and the time-integrated radiative forcing $\int F d \tau$ is not expected to hold for any climate change scenario; it holds only for steadily increasing forcing. Deviations from the linear relationship are found during years that are affected by intense explosive volcanic eruptions when the forcing suddenly drops. During these years, the GMTSL shows a smaller response to changes in the climate radiative forcing than it does at long time scales in response to greenhouse gas and aerosol emissions. It is not clear whether this is because GMTSL responds differently to rapid changes in the climate radiative forcing or because GMTSL responds to volcanic emissions (emissions of sulfate aerosols in the high troposphere and in the stratosphere) in a different manner than it

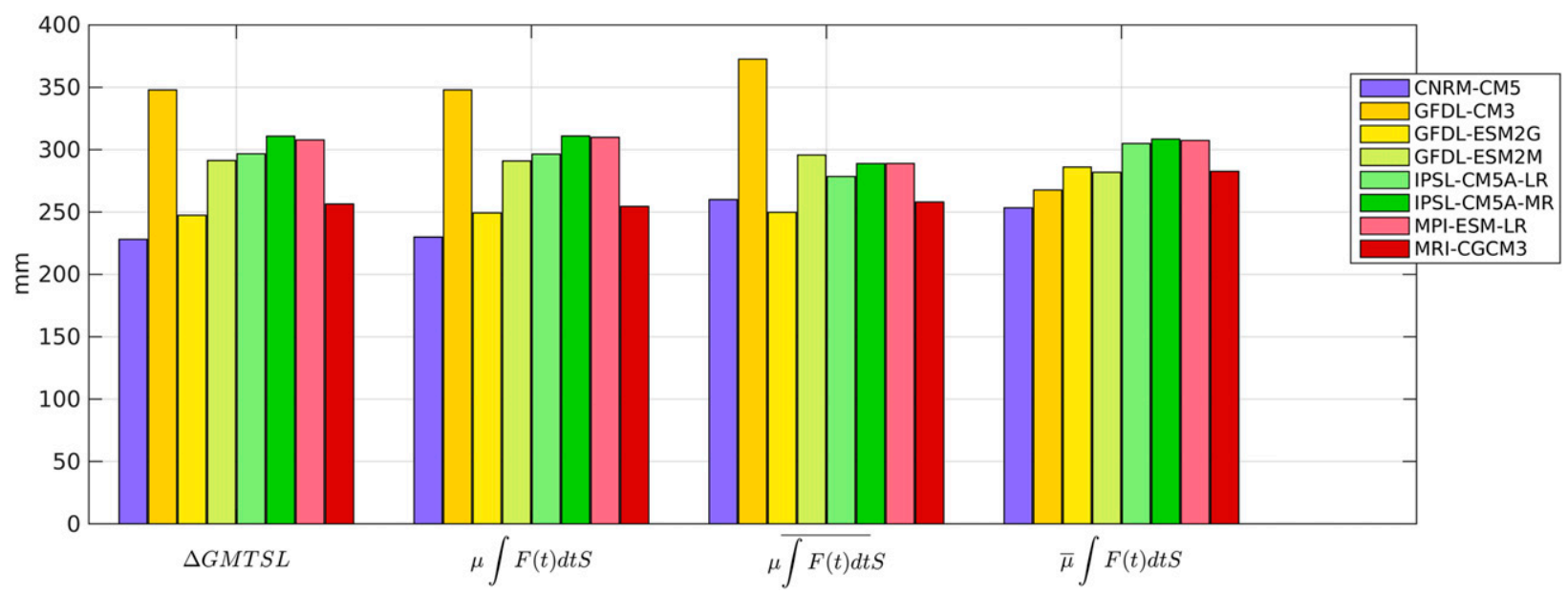

FIG. 15. Climate model global mean thermosteric sea level rise in 2099 relative to $2006(\mathrm{~mm})$ under the RCP8.5 scenario computed from the $3 \mathrm{D}$ temperature and salinity fields (first group), from the climate coefficient relationship $\mu \int F(t) d t S$ (second group), from the climate coefficient relationship using the model ensemble mean values for $\int F(t) d t$ (third group), and for $\mu$ (fourth group). The overbar denotes an averaging across the model ensemble. Only models conserving energy ( $\beta$ within $20 \%$ of 0.93 ) are included. 


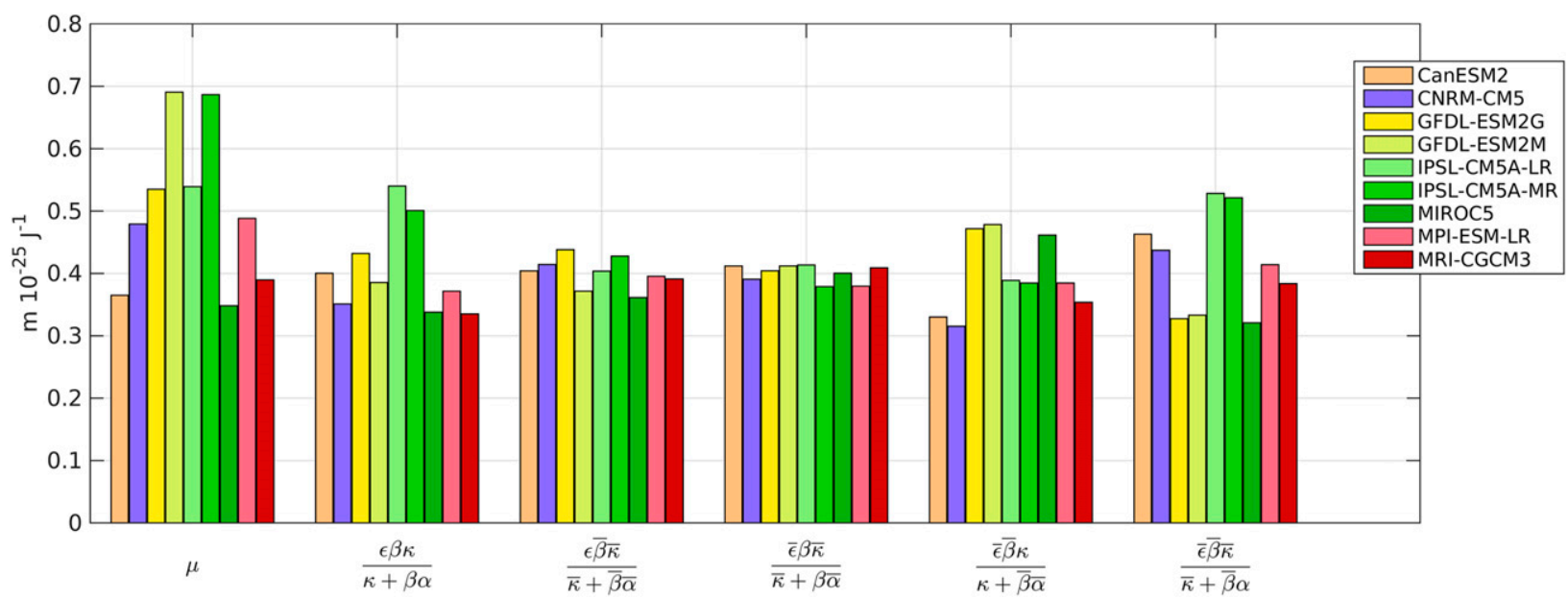

FIG. 16. Twentieth-century values $\left(10^{-25} \mathrm{~m} \mathrm{~J}^{-1}\right)$ of the transient thermosteric sea level response of the climate system $\mu$ (first group), of $\epsilon \beta \kappa /(\kappa+\beta \alpha)$ (second group), of $\epsilon \beta \kappa /(\kappa+\beta \alpha)$ when only $\epsilon$ varies across models (third group), of $\epsilon \beta \kappa /(\kappa+\beta \alpha)$ when only $\beta$ varies across models (fourth group), of $\epsilon \beta \kappa /(\kappa+\beta \alpha)$ when only $\kappa$ varies across models (fifth group), and of $\epsilon \beta \kappa /(\kappa+\beta \alpha)$ when only $\alpha$ varies across models (sixth group). The overbar denotes an averaging across the model ensemble.

does to anthropogenic emissions (emissions of tropospheric greenhouse gases and aerosols).

We also note small deviations from the linear relationship at the end of the twenty-first-century simulations; when the radiative forcing increases, the GMTSL tends to rise slightly slower. This is due to a deceleration of the global ocean heat uptake as the forcing increases. Examining the ocean heat uptake efficiency $\kappa$ and the surface temperature changes $\Delta T$ shows that $\kappa$ tends to slightly decrease as the forcing increases for all models, which means that the global ocean heat uptake decelerates. If this behavior, which is robust across models, is confirmed in the real world, this would mean that the transient thermosteric sea level response of the climate system tends to decrease as the forcing increases, and projections of future sea level based on a linear equation such as (7) tend to overestimate future thermosteric sea level rise when the radiative forcing increases with time.

Acknowledgments. The authors acknowledge the World Climate Research Programme's Working Group on Coupled Modelling, which is responsible for CMIP, and we thank the climate modeling groups (models listed in Table 1 of this paper) for producing and making available their model output. For CMIP the U.S. Department of Energy's Program for Climate Model Diagnosis and Intercomparison provides coordinating

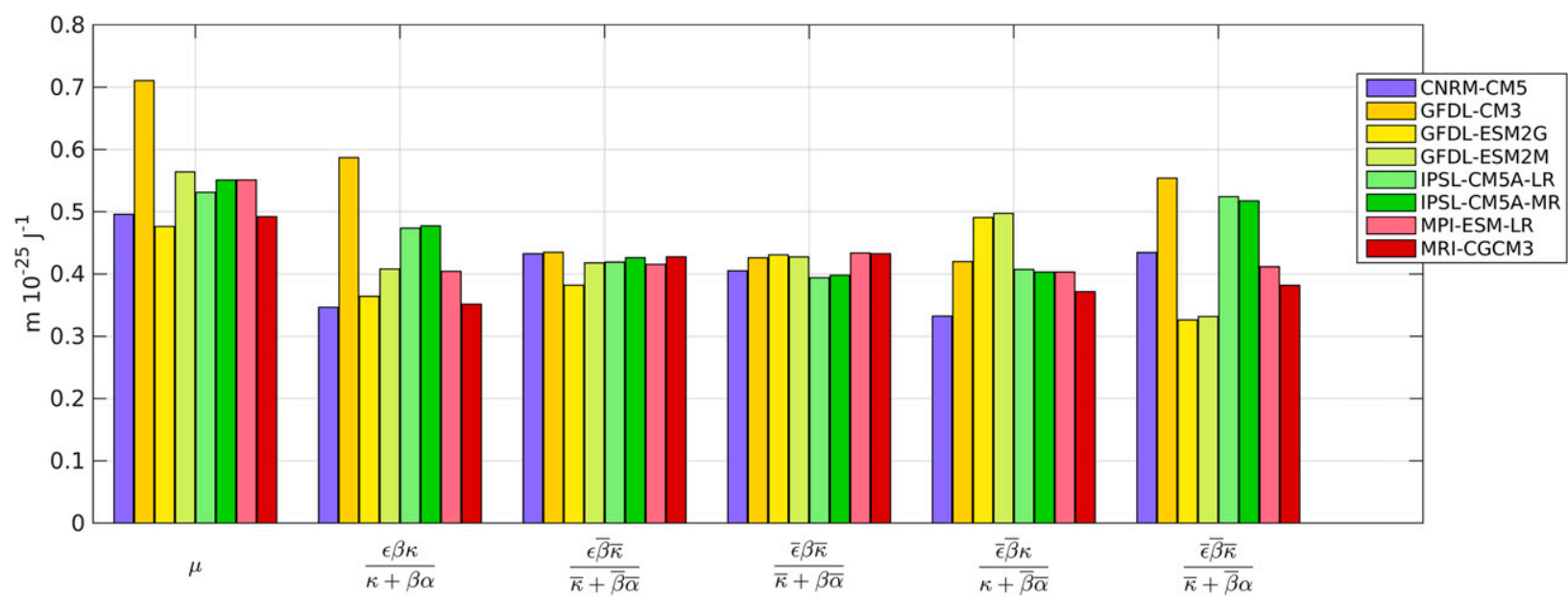

FIG. 17. Twenty-first-century (under the RCP8.5 scenario) values $\left(10^{-25} \mathrm{~m} \mathrm{~J}^{-1}\right)$ of the transient thermosteric sea level response of the climate system $\mu$ (first group), of $\epsilon \beta \kappa /(\kappa+\beta \alpha)$ (second group), of $\epsilon \beta \kappa /(\kappa+\beta \alpha)$ when only $\epsilon$ varies across models (third group), of $\epsilon \beta \kappa /(\kappa+$ $\beta \alpha$ ) when only $\beta$ varies across models (fourth group), of $\epsilon \beta \kappa /(\kappa+\beta \alpha)$ when only $\kappa$ varies across models (fifth group), and of $\epsilon \beta \kappa /(\kappa+\beta \alpha)$ when only $\alpha$ varies across models (sixth group). The overbar denotes an averaging across the model ensemble. 
support and led development of software infrastructure in partnership with the Global Organization for Earth System Science Portals. The authors thank the International Space Science Institute (ISSI; Bern, Switzerland) for support of the International Team on contemporary regional and global sea level rise.

\section{APPENDIX A}

\section{Ocean Heat Uptake Efficiency}

The climate resistance $\rho$ verifies the following equation: $\rho=F / \Delta T=\alpha+N / \Delta T$, which can be rewritten as $N / \Delta T=\rho-\alpha$. In the literature, $N$ is generally identified as the heat flux entering the ocean (because the earth energy imbalance is essentially stored in the ocean in the form of heat) so that $\rho-\alpha$ is identified as the ocean heat uptake efficiency $\kappa$ (e.g., Gregory and Forster 2008). In this study, we consider the fraction of the earth energy imbalance $\beta$ that enters the ocean (section 3a) such that the heat flux that actually enters the ocean is rather $\beta N$. Therefore, $\rho-\alpha$ is identified here as $\kappa / \beta$.

$$
\operatorname{var}(\Delta \mathrm{GMTSL})=\sum_{i}\left(\frac{\partial \Delta \mathrm{GMTSL}}{\partial x_{i}}\right)^{2} \operatorname{var}\left(x_{i}\right)+\sum_{i} \sum_{j \neq i}\left(\frac{\partial \Delta \mathrm{GMTSL}}{\partial x_{i}}\right)\left(\frac{\partial \Delta \mathrm{GMTSL}}{\partial x_{j}}\right) \operatorname{cov}\left(x_{i}, x_{j}\right) .
$$

The fraction of across-model variance in $\triangle$ GMTSL due to the across-model variance in $x_{i}$ is therefore given by

$$
\left(\frac{\partial \Delta \mathrm{GMTSL}}{\partial x_{i}}\right)^{2} \operatorname{var}\left(x_{i}\right) / \operatorname{var}(\Delta \mathrm{GMTSL}) .
$$

For instance, considering (6), the across-model variance in $\Delta$ GMTSL (between $t_{\mathrm{ref}}$ and $t_{\mathrm{end}}$ ) explained by the across-model variance in $\epsilon$ is given by

$$
\left(S \beta \frac{\kappa}{\kappa+\beta \alpha} \int_{t_{\text {ref }}}^{t_{\text {end }}} F(\tau) d \tau\right)^{2} \operatorname{var}(\epsilon) / \operatorname{var}(\Delta \mathrm{GMTSL}) .
$$

\section{REFERENCES}

Ablain, M., and Coauthors, 2014: Improved sea level record over the satellite altimetry era (1993-2010) from the Climate Change Initiative Project. Ocean Sci. Discuss., 11, 2029-2071, doi:10.5194/osd-11-2029-2014.

Abraham, J. P., and Coauthors, 2013: A review of global ocean temperature observations: Implications for ocean heat content estimates and climate change. Rev. Geophys., 51, 450-483, doi:10.1002/rog.20022.

Andrews, T., J. M. Gregory, M. J. Webb, and K. E. Taylor, 2012: Forcing, feedbacks and climate sensitivity in CMIP5 coupled atmosphere-ocean climate models. Geophys. Res. Lett., 39, L09712, doi:10.1029/2012GL051607.

Bouttes, N., J. M. Gregory, and J. A. Lowe, 2013: The reversibility of sea level rise. J. Climate, 26, 2502-2513, doi:10.1175/ JCLI-D-12-00285.1.
Bryden, H., 1973: New polynomials for thermal expansion, adiabatic temperature gradient and potential temperature of sea water. Deep-Sea Res., 20, 401-408.

Cazenave, A., H.-B. Dieng, B. Meyssignac, K. von Shuckmann, B. Decharme, and E. Berthier, 2014: The rate of sea-level rise. Nat. Climate Change, 4, 358-361, doi:10.1038/nclimate2159.

Church, J. A., N. J. White, and J. Arblaster, 2005: Significant decadalscale impact of volcanic eruptions on sea level and ocean heat content. Nature, 438, 74-77, doi:10.1038/nature04237.

— , and Coauthors, 2011: Revisiting the Earth's sea-level and energy budgets from 1961 to 2008. Geophys. Res. Lett., 38, L18601, doi:10.1029/2011GL048794.

— 2013: The Physical Science Basis, T. F. Stocker et al., Cambridge University Press, 1137-1216. [Available online at https:// www.ipcc.ch/pdf/assessment-report/ar5/wg1/WG1AR5_Chapter13_ FINAL.pdf.]

D. Monselesan, J. M. Gregory, and B. Marzeion, 2013b: Evaluating the ability of process based models to project sea-level change. Environ. Res. Lett., 8, 014051, doi:10.1088/ 1748-9326/8/1/014051.

Collins, M., and Coauthors, 2013: Long-term climate change: Projections, commitments and irreversibility. Climate Change 2013: The Physical Science Basis, T. F. Stocker et al., Cambridge University Press, 1029-1136.

Collins, W. D., and Coauthors, 2006: Radiative forcing by wellmixed greenhouse gases: Estimates from climate models in the Intergovernmental Panel on Climate Change (IPCC) Fourth Assessment Report (AR4). J. Geophys. Res., 111, D14317, doi:10.1029/2005JD006713.

Delworth, T. L., V. Ramaswamy, and G. L. Stenchikov, 2005: The impact of aerosols on simulated global ocean temperature and heat content in the 20th century. Geophys. Res. Lett., 32, L24709, doi:10.1029/2005GL024457.

Domingues, C. M., J. A. Church, N. J. White, P. J. Gleckler, S. E. Wijffels, P. M. Barker, and J. R. Dunn, 2008: Improved estimates 
of upper-ocean warming and multi-decadal sea-level rise. Nature, 453, 1090-1093, doi:10.1038/nature07080.

Durack, P. J., P. J. Gleckler, F. W. Landerer, and K. E. Taylor, 2014: Quantifying underestimates of long-term upper-ocean warming. Nat. Climate Change, 4, 999-1005, doi:10.1038/nclimate2389.

Forster, P. M., T. Andrews, P. Good, J. M. Gregory, L. S. Jackson, and M. Zelinka, 2013: Evaluating adjusted forcing and model spread for historical and future scenarios in the CMIP5 generation of climate models. J. Geophys. Res., 118, 1139-1150, doi:10.1002/jgrd.50174.

Gregory, J. M., 2010: Long-term effect of volcanic forcing on ocean heat content. Geophys. Res. Lett., 37, L22701, doi:10.1029/ 2010 GL045507.

_ volcanic forcing underestimate historical ocean thermal expansion. Geophys. Res. Lett., 40, 1600-1604, doi:10.1002/grl.50339.

, and Coauthors, 2013b: Twentieth-century global-mean sealevel rise: Is the whole greater than the sum of the parts? J. Climate, 26, 4476-4499, doi:10.1175/JCLI-D-12-00319.1.

- and P. M. Forster, 2008: Transient climate response estimated from radiative forcing and observed temperature change. J. Geophys. Res., 113, D23105, doi:10.1029/2008JD010405.

— , and M. J. Webb, 2008: Tropospheric adjustment induces a cloud component in $\mathrm{CO}_{2}$ forcing. J. Climate, 21, 58-71, doi:10.1175/2007JCLI1834.1.

—- and Coauthors, 2004: A new method for diagnosing radiative forcing and climate sensitivity. Geophys. Res. Lett., 31, L03205, doi:10.1029/2003GL018747.

_ J. J. Aowe, and S. F. B. Tett, 2006: Simulated global-mean sea level changes over the last half-millennium. J. Climate, 19, 4576-4591, doi:10.1175/JCLI3881.1.

Hallberg, R. W., A. Adcroft, J. P. Dunne, J. P. Krasting, and R. J. Stouffer, 2013: Sensitivity of twenty-first-century global-mean steric sea level rise to ocean model formulation. J. Climate, 26, 2947-2956, doi:10.1175/JCLI-D-12-00506.1.

Ishii, M., and M. Kimoto, 2009: Reevaluation of historical ocean heat content variations with time-varying XBT and MBT depth bias corrections. J. Phys. Oceanogr., 65, 287-299, doi:10.1007/s10872-009-0027-7.

Kuhlbrodt, T., and J. M. Gregory, 2012: Ocean heat uptake and its consequences for the magnitude of sea level rise and climate change. Geophys. Res. Lett., 39, L18608, doi:10.1029/ 2012GL052952.
Levitus, S., J. I. Antonov, and T. P. Boyer, 2005: Warming of the world ocean, 1955-2003. Geophys. Res. Lett., 32, L02604, doi:10.1029/2004GL021592.

,,-- , R. A. Locarnini, H. E. Garcia, and A. V. Mishonov, 2009: Global ocean heat content 1955-2008 in light of recently revealed instrumentation problems. Geophys. Res. Lett., 36, L07608, doi:10.1029/2008GL037155.

_ - and Coauthors, 2012: World ocean heat content and thermosteric sea level change (0-2000 m), 1955-2010. Geophys. Res. Lett., 39, L10603, doi:10.1029/2012GL051106.

Llovel, W., I. Fukumori, and B. Meyssignac, 2013: Depth-dependent temperature change contributions to global mean thermosteric sea level rise from 1960 to 2010. Global Planet. Change, 101, 113-118, doi:10.1016/j.gloplacha.2012.12.011.

Moss, R. H., and Coauthors, 2010: The next generation of scenarios for climate change research and assessment. Nature, 463, 747756, doi:10.1038/nature08823.

Palmer, M. D., and D. McNeall, 2014: Internal variability of Earth's energy budget simulated by CMIP5 climate models. Environ. Res. Lett., 9, 034016, doi:10.1088/1748-9326/9/3/ 034016.

Purkey, S. G., and G. C. Johnson, 2010: Warming of global abyssal and deep Southern Ocean waters between the 1990s and 2000s: Contributions to global heat and sea level rise budgets. J. Climate, 23, 6336-6351, doi:10.1175/2010JCLI3682.1.

Russell, G. L., V. Gornitz, and J. R. Miller, 2000: Regional sea-level changes projected by the NASA/GISS atmosphere-ocean model. Climate Dyn., 16, 789-797, doi:10.1007/s003820000090.

Sen Gupta, A., N. C. Jourdain, J. N. Brown, and D. Monselesan, 2013: Climate drift in the CMIP5 models. J. Climate, 26, 85978615, doi:10.1175/JCLI-D-12-00521.1.

Taylor, K. E., R. J. Stouffer, and G. A. Meehl, 2012: An overview of CMIP5 and the experiment design. Bull. Amer. Meteor. Soc., 93, 485-498, doi:10.1175/BAMS-D-11-00094.1.

Webb, M., and Coauthors, 2006: On the contribution of local feedback mechanisms to the range of climate sensitivity in two GCM ensembles. Climate Dyn., 27, 17-38, doi:10.1007/s00382-006-0111-2.

Wong, P., I. J. Losada, J. P. Gattuso, J. Hinkel, A. Khattabi, K. L. McInnes, Y. Saito, and A. Sallenger, 2013: Coastal systems and low-lying areas. Climate Change 2014: Impacts, Adaptation, and Vulnerability, C. B. Field et al., Eds., Cambridge University Press, 361-409. [Available online at https://ipcc-wg2.gov/AR5/ images/uploads/WGIIAR5-Chap5_FINAL.pdf.] 